SERVIÇO DE PÓS-GRADUAÇÃO DO ICMC-USP

Data de Depósito: 26/05/2008

Assinatura:

\title{
Triangulações regulares e aplicações
}

\author{
Fernando Bissi Pires
}

Orientador: Prof. Dr. Luis Gustavo Nonato

Dissertação apresentada ao Instituto de Ciências Matemáticas e de Computação - ICMC-USP, como parte dos requisitos para obtenção do título de Mestre em Ciências - Ciências de Computação e Matemática Computacional.

USP - São Carlos

Maio/2008 



\section{Agradecimentos}

Ao meu orientador, Prof. Dr. Luis Gustavo Nonato, pelo apoio, sugestões e profissionalismo na orientação durante todo o período deste trabalho.

Aos meus familiares pelo amor, incentivo e constante apoio durante toda minha vida.

Aos professores e funcionários do ICMC, pela disposição e atenção.

À FAPESP pela bolsa de estudos. 



\section{Resumo}

A triangulação de Delaunay de um conjunto de pontos é uma importante entidade geométrica cujas aplicações abrangem diversas áreas da ciência. Triangulações regulares, que podem ser vistas como uma generalização da triangulação de Delaunay, onde pesos são associados aos vértices, também têm sido aplicadas em diversos problemas como reconstrução a partir de nuvens de pontos [5], geração de malha [12], modelagem molecular [7] e muitos outros. Apesar de ser muito utilizada, a fundamentação teórica referente à triangulação regular ainda não está tão desenvolvida quanto para triangulação de Delaunay. Por exemplo, pouco se sabe a respeito da dinâmica de uma triangulação regular [22] quando os pesos associados aos vértices mudam.

Este trabalho tem como objetivo principal desenvolver um arcabouço teórico e computacional que permita representar uma triangulação qualquer como uma triangulação regular. Para isso, um estudo da dinâmica das operações de flip frente à variação de pesos nos vértices deve ser realizado. Este estudo tem como base o mapeamento da triangulação em um politopo que define os possíveis pesos para os vértices. Tal politopo pode ser obtido por meio de um sistema de inequações que gera um problema de programação linear cuja solução fornece os pesos adequados.

A transformação de uma triangulação qualquer em triangulação regular permite o desenvolvimento de novas técnicas de morphing entre malhas e algoritmos para modelar níveis de detalhe, sendo este mais um objetivo deste trabalho. 



\section{Abstract}

Delaunay triangulation of a set of points is an important geometrical entity whose applications encompass a range of scientific fields. Regular triangulations, which can be seen as a generalization of Delaunay triangulation where weights are assigned to vertices, have also been widely employed in several problems, as for example mesh reconstruction from point clouds [5], mesh generation [12] and molecular modelling [7]. In spite of their applicability, the theoretical background of regular triangulations is not so developed as the theory of Delaunay triangulation. For example, the dynamic of regular triangulation is not completely known when the vertices weights change [22].

This work aims at developing a computational and theoretical framework that allow to represent a given triangulation as a regular triangulation. In this context, an investigation into the dynamic of edge flip operations regarding changes in the vertices weight must be accomplished. This investigation is based on mapping the triangulation in a polytope that defines the space of vertices weights. Such polytope can be built from an inequation system that can be associate to a linear program problem whose solution supplies the appropriated weights.

By representing a triangulation as a regular triangulation one can conceive a new mesh morphing scheme and level of detail algorithm, being this another goal of this work. 



\section{Sumário}

Agradecimentos $\quad$ i

Resumo iii

$\begin{array}{lll}\text { Abstract } & \text { v }\end{array}$

1 Introdução 1

2 Conceitos Básicos 5

2.1 Complexo Simplicial e Triangulação Regular . . . . . . . . . . . . . . . 5

2.2 Triangulação Regular em Superfícies . . . . . . . . . . . . . . . . . 12

2.3 Diagrama de Voronoi . . . . . . . . . . . . . . . . . 13

3 Algoritmos $\quad 17$

3.1 Triangulação Regular . . . . . . . . . . . . . . . . . . . . 17

3.1.1 Algoritmo Incremental com Flip . . . . . . . . . . . . . . . . . . 17

3.1.2 Triangulações Dinâmicas . . . . . . . . . . . . . . . . . . . . . 19

3.1 .3 Hiperplano Associado . . . . . . . . . . . . . . . . . 20

3.2 Diagrama de Voronoi com Peso . . . . . . . . . . . . . . . . . . 21

3.2.1 Algoritmo Sweepline . . . . . . . . . . . . . . . . . . . 23

4 Desenvolvimentos Realizados $\quad 25$

4.1 Prevendo um flip . . . . . . . . . . . . . . . . . . 25

4.2 Algoritmo para Triangulações Dinâmicas . . . . . . . . . . . . . . . . . . 31

4.3 Calculando Pesos em Duas Dimensões . . . . . . . . . . . . . . . . 32 
4.4 Três Dimensões . . . . . . . . . . . . . . . . . . . . . . . 37

4.5 Triangulações não Convexas . . . . . . . . . . . . . . . . . . . . . . . . . 39

5 Aplicações $\quad 41$

5.1 Compressão de Malhas . . . . . . . . . . . . . . . . . . . . . . . . . . 41

5.2 Nível de Detalhes . . . . . . . . . . . . . . . . . . . . . . . 45

5.3 Morphing de Malhas . . . . . . . . . . . . . . . . . . . . 47

6 Conclusões $\quad 53$

$\begin{array}{ll}\text { Bibliografia } & 55\end{array}$ 


\section{Lista de Figuras}

2.1 Representação de uma aresta $e$ localmente Delaunay. . . . . . . . . . . . 7

2.2 Representação geométrica da distância com peso. . . . . . . . . . . . . . 7

2.3 Seqüencia de simplexos interceptados pelo segmento $x v \ldots \ldots \ldots$. . . . 8

2.4 Possíveis flips em uma triangulação com 2 dimensões. . . . . . . . . . . . . 8

2.5 As duas primeiras projeções correspondem aos flips $1-3$ e $3-1$, quando se insere ou remove um ponto. A terceira projeção corresponde ao flip $2-2 . \quad 9$

2.6 Ilustra um pequeno exemplo de triangulação Regular. . . . . . . . . . . . . 10

2.7 Exemplo onde ocorre um flip $3-1$ no ponto $\hat{p}_{1}$ ao inserir o ponto com peso

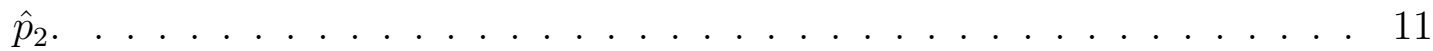

2.8 Possíveis flips em uma triangulação com 3 dimensões. . . . . . . . . . . . . . 11

2.9 Todas as arestas são aresta de Voronoi do diagrama de Voronoi deste conjunto de pontos em $\mathbb{R}^{2}$, a união de cada três arestas é um vértice de Voronoi. 14

2.10 Simples exemplo de um power diagrama. . . . . . . . . . . . . . . 15

3.1 Politopo de Schönhardt, contra exemplo para o algoritmo de flip. . . . . . 18

3.2 Neste exemplo temos $\hat{p} \in \mathbb{R}^{2}$ cujo plano $\eta_{p}$ se intercepta no parabolóide $\|x\|^{2}$. 21

3.3 Neste exemplo o ponto $s_{i}$ tem seu peso alterado. Enquanto não há alteração na relação de incidência entre as faces do envelope superior, nenhum flip é realizado na triangulação, como mostra a figura a) e b). A mudança nas faces do envelope superior indica um flip de arestas na triangulação, como

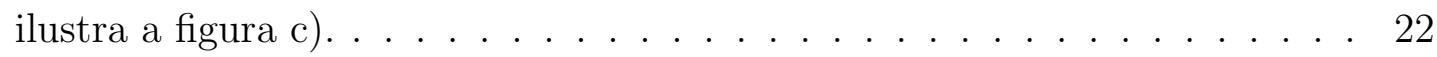


3.4 Inicialmente temos uma reta vertical passando pelos pontos, criando as respectivas parábolas. Na imagem a) houve apenas intersecções entre duas parábolas criando segmentos de retas, na imagem b) houve intersecção entre as três parábolas entre os três pontos azuis superiores criando um vértice de Voronoi. Nas demais imagens a reta continua movendo até que todos os vértices estejam a sua esquerda e não haja mais intersecções entre 3 parábolas. . . . . . . . . . . . . . . . . . . . 23

4.1 Em a) o conjunto $P$ e sua respectiva triangulação $T$, e em b) temos a triangulação Delaunay de $S$, note que a triangulação $T$ não é Delaunay. 34

4.2 A triangulação de $P$ com os pesos $W$ associados a eles, e se obtém a triangulação $T$ como regular. . . . . . . . . . . . . . . . . . . . . . . . 35

4.3 Uma triangulação cujas arestas não são localmente Delaunay. . . . . . . . . 36

4.4 Triangulação regular e pesos dos vértices da imagem $4.3 . \quad \ldots$. . . . . . 36

4.5 Configuração de triângulos estudada. . . . . . . . . . . . . . . . . . 37

4.6 A imagem a) é uma triangulação não convexa, em b) foram escolhidos três vértices do bordo cujo triângulo formado por eles pertence ao interior da triangulação em c) e d) os triângulos formados pelos vértices do bordo pertencem ao exterior e serão adicionados à triangulação. . . . . . . . . . . . 40

4.7 Nesta imagem, uma triangulação não convexa torna-se convexa utilizando o esquema descrito e pontos de controle são adicionados. Neste caso apenas quatro pontos de controles foram necessários. . . . . . . . . . . . . . . . . 40

5.1 Plano de subdivisão de uma região, imagem retirada de [25] . . . . . . . . . 43

5.2 Exemplo com alguns níveis de subdivisão gerados pela BSP, imagem reti-

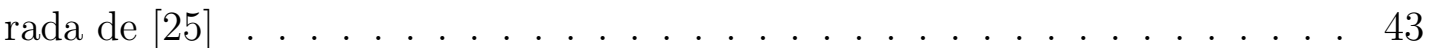

5.3 Na primeira imagem temos uma superfície homeomorfa a um disco, que na imagem segundo é parametrizada em um domínio convexo bidimensional, cujos pesos que a tornam uma triangulação regular São mostrados na terceira imagem. . . . . . . . . . . . . . . . . . . . . 46 
5.4 Exemplo de resultados de níveis de refinamento obtidos com este esquema, em ambos os casos os pontos em destaque foram escolhidos pelo usuário. 47

5.5 Exemplo de como dividir malhas em regiões e correspondê-las entre duas malhas. . . . . . . . . . . . . . . . . . . . . 48

5.6 Em a) uma triangulação foi dividida em duas regiões, em b) um vértice do bordo foi removido de uma das regiões criando um buraco em c) o vértice foi removido simultaneamente em ambas as regiões. . . . . . . . . . . . . . 49

5.7 Dois exemplos de morphing entre malhas obtidos através de interpolações entre triangulações regulares. . . . . . . . . . . . . . . . . . . 51 



\section{Capítulo 1}

\section{Introdução}

A triangulação de Delaunay de um conjunto $P$ de pontos é a triangulação cujo circuncírculo de cada triângulo não possui nenhum ponto de $P$ no seu interior. Esta triangulação é muito usada em várias áreas. Dentre os motivos que justificam tamanha popularidade estão as boas propriedades da triangulação de Delaunay, como por exemplo a maximização do ângulo mínimo no caso bidimensional, isto é, dentre todas as triangulações de $P$, a triangulação de Delaunay maximiza o menor ângulo da triangulação, tendendo a gerar triângulos de boa qualidade. Quando se associa a cada ponto um escalar e estende-se o conceito de círculo vazio para os chamados ortocírculos, dá-se origem à triangulação de Delaunay com peso, ou triangulação regular. Apesar de ser muito utilizada, a fundamentação teórica referente à triangulação regular ainda não está tão desenvolvida quanto para triangulação de Delaunay.

A operação de flip é a base dos algoritmos para construção de uma triangulação regular. Inicialmente, a idéia de flip foi introduzida por Lawson (1972) [17] que se difundiu e foi utilizada por muitos outros autores, não apenas em triangulações regulares. Utilizando esta idéia é possível alterar pesos de triângulos dinamicamente, apesar de que para fazer isto a maioria dos algoritmos remove o ponto cujo peso foi alterado e insere o ponto com o novo peso. Atualmente existem vários algoritmos para construção de uma triangulação regular, dentre eles destaca-se o algoritmo de flip incremental [9], que constrói a triangulação regular a partir de uma triangulação regular inicial e para cada ponto com peso inserido realiza uma seqüencia de flips que mantém as propriedades de triangulação 
regular.

A triangulação de Delaunay tem como dual o diagrama de Voronoi. Tal diagrama divide o espaço em regiões contendo os pontos de $P$ tal que cada ponto de $P$ esta contido em apenas uma região. Assim como a triangulação de Delaunay, o diagrama de Voronoi tem sido aplicado em diversos problemas. Um exemplo típico é o dos correios, onde a área de atuação de cada agência precisa ser determinada. Considerando que as agências são idênticas, o diagrama de Voronoi das mesmas fornece as regiões de abrangência desejadas. Porém, se as agências tiverem um número diferente de funcionários é de se esperar que a agência com mais funcionários possua uma região de abrangência maior. Neste caso, podese associar a cada um dos pontos (agências) um peso (número de funcionários) que irá alterar o diagrama e conseqüentemente as regiões de abrangência. Este novo diagrama é conhecido como power diagrama, ou diagrama de Voronoi com peso, que possui como dual a triangulação regular. Quando todos os pesos dos pontos são iguais então a triangulação regular será a triangulação de Delaunay e o power diagrama será o diagrama de Voronoi.

Este trabalho tem como objetivo principal desenvolver um mecanismo que torne uma triangulação qualquer em triangulação regular. Tal transformação será feita por meio de atribuição de pesos aos vértices da triangulação. A partir dos pesos e das coordenadas dos vértices pode-se recuperar a triangulação original por meio de um algoritmo para construção de uma triangulação regular. Transformando o problema de cálculo dos pesos para um problema de cálculo de intersecção de hiperplanos (semi-espaços) pode-se gerar um sistema de inequações que quando resolvido fornece os pesos adequados. Para resolver tal problema utiliza-se otimização linear, onde a função objetivo maximiza o número de pesos iguais a zero. Uma vez calculados os pesos (objetivando que a maioria destes sejam iguais a zero) pode-se desconsiderar as relações de conectividade no armazenamento da malha, pois esta poderá ser recuperada por meio dos algoritmos de construção das triangulações regulares. Este fato motiva o uso de nossa proposta no problema de compressão de malhas.

Compressão de malha consiste em armazenar e representar uma malha com o mínimo possível de informações que possibilite a reconstrução fiel desta. Em geral, o problema 
de compressão de malha é dividido em duas partes: compressão de conectividade e compressão das coordenadas dos pontos. A maior compressão se dá na compressão das coordenadas dos pontos, onde em geral são feitas quantizações. Basicamente existem duas formas para realizar uma compressão, por simplificações ou divisão espacial, tendo este, segundo [25], até o momento apresentando melhores resultados.

Este trabalho também foi aplicado em nível de refinamento e morphing de malhas. Nível de refinamento consiste em determinar malhas mais simples a partir de uma malha inicial. É proposto tornar a malha inicial uma triangulação regular e remover os pontos tornando-os redundantes. Morphing de malha consiste em encontrar malhas intermediárias entre duas malhas $A$ e $B$ de tal forma que as malhas intermediárias transformem a malha $A$ na malha $B$. Usando triangulação regular basta determinar uma função que leve os pontos e os pesos de $A$ aos pontos e pesos de $B$ e as malhas intermediárias serão automaticamente obtidas.

No Capítulo 2 deste trabalho estão definidos os conceitos básicos que leva à definição da triangulação de Delaunay e em sua generalização para triangulações regulares em $\mathbb{R}^{n}$, também é definido seu dual geométrico, o diagrama de Voronoi com peso, e triangulações regulares em superfícies. No Capítulo 3 é feito um levantamento bibliográfico detalhando a descrição do algoritmo de flip incremental [9], uma estratégia para trabalhar dinamicamente com os pesos em uma triangulação regular [22], a definição de hiperplano [7] que é a base para nosso estudo e também o algoritmo sweepline [13] para o cálculo de um diagrama de Voronoi com peso. No Capítulo 4 está descrita a técnica que desenvolvemos capaz de transformar uma triangulação qualquer em triangulação regular, assim como um algoritmo para triangulações regulares dinâmicas. Tal técnica baseia-se em um sistema de inequações cuja solução determina os pesos, este sistema de inequações é resolvido utilizando programação linear. No capítulo 5 são descritos três possíveis aplicações para este problema. Finalmente no capítulo 6, as conclusões adquiridas com este estudo são apresentadas. 



\section{Capítulo 2}

\section{Conceitos Básicos}

Neste capítulo introduzimos os conceitos básicos que nos levam a uma definição de triangulação de Delaunay, triangulação regular e seus respectivos duais, diagrama de Voronoi e diagrama de Voronoi com peso.

\subsection{Complexo Simplicial e Triangulação Regular}

Definição 2.1.1. Um conjunto de pontos $P=\left\{p_{0}, p_{1}, \ldots, p_{m}\right\} \in \mathbb{R}^{n}$, com $m \leq n$, é dito linearmente independente se os vetores $p_{0}-p_{1}, p_{0}-p_{2}, \ldots, p_{0}-p_{m}$ forem linearmente independentes.

Definição 2.1.2. Dado um conjunto de pontos linearmente independente $P=\left\{p_{0}, \ldots, p_{m}\right\}$ $\in \mathbb{R}^{n}$, um $m$-1-simplexo $\sigma$ gerado por $P$, denotado por $\sigma=\left[p_{0}, \ldots p_{m}\right]$ é o conjunto de todos os pontos gerados como combinação linear convexa de $P$, ou seja, $\sigma=\left\{p \in \mathbb{R}^{n} \mid p=\right.$ $\left.\sum \lambda_{i} p_{i}, \sum \lambda_{i}=1, \lambda_{i} \geq 0\right\}$

Em $\mathbb{R}^{n}$ o maior número de pontos linearmente independentes é $n+1$, logo em $\mathbb{R}^{n}$ temos simplexos de dimensão $0,1,2, \ldots, n$. Onde 0 -simplexos são vértices, 1 -simplexos são arestas, 2-simplexos são triângulos e assim por diante.

Dado um simplexo $\sigma=\left[p_{0}, \ldots p_{m}\right]$ qualquer simplexo gerado a partir de um subconjunto de pontos de $\left\{p_{0}, p_{1}, \ldots, p_{m}\right\}$ é chamado de face de $\sigma$.

Definição 2.1.3. Um complexo simplicial $K$ é uma coleção de simplexos satisfazendo: 
1. Se $\sigma \in K$ então todas as faces de $\sigma$ estão em $K$.

2. Se $\sigma$ e $\gamma \in K$ e $\sigma \cap \gamma \neq 0$ então $\sigma \cap \gamma$ é uma face em comum de $\sigma$ e $\gamma$.

A dimensão de um complexo simplicial $K$ é a maior dimensão de seus simplexos.

Seja $\sigma \in K$ um simplexo, a estrela de $\sigma$, denotada por $s t(\sigma)$ é o conjunto de todos os simplexos contidos em $K$ que contém $\sigma$ como uma de suas faces. O link de $\sigma, l k(\sigma)$, é o conjunto de todas faces dos simplexos pertencentes a $s t(\sigma)$ que não possuem intersecção $\operatorname{com} \sigma$.

Definição 2.1.4. Um conjunto de pontos $P$ é dito estar em posição geral se não existe nenhum sub-espaço afim de $\mathbb{R}^{n}$ contendo $P$.

Definição 2.1.5. O fecho convexo de conjunto de pontos $P$ é o conjunto de todas as combinações lineares convexas dos pontos de $P$, ou seja, o fecho convexo de $P$ é o polígono convexo de menor volume que contém todos os pontos de $P$.

Definição 2.1.6. Sejam $p_{0}, p_{1}, p_{2}, \ldots, p_{n}, n+1$ pontos em $\mathbb{R}^{n}$, que formam o $n$-simplexo $\sigma$, definimos como circunsfera de $\sigma$ a esfera que passa por todos os vértices de $\sigma$.

Definição 2.1.7. Dado um conjunto de pontos em posição geral $P=\left\{p_{0}, p_{1}, \ldots, p_{m}\right\} \in$ $\mathbb{R}^{n}$, a triangulação de Delaunay de $P$ é um n-complexo simplicial $K$ satisfazendo:

1. Todo ponto $p_{i}$ é vértice de um n-simplexo de $K$.

2. A união dos simplexos em $K$ gera o fecho convexo de $P$.

3. A circunsfera de todos os n-simplexos é vazia de pontos, ou seja, não existe nenhum ponto de $P$ no interior das circunsferas dos n-simplexos.

Se apenas as condições 1 e 2 forem satisfeitas $K$ é dita uma triangulação.

Definição 2.1.8. Uma aresta e de uma triangulação $K$ é dita localmente Delaunay se:

1. e pertence a apenas um triângulo e está no bordo do fecho convexo.

2. e pertence a dois triângulos abc e abd e o vértice d está fora do circuncírculo de abc, como ilustra a imagem 2.1. 


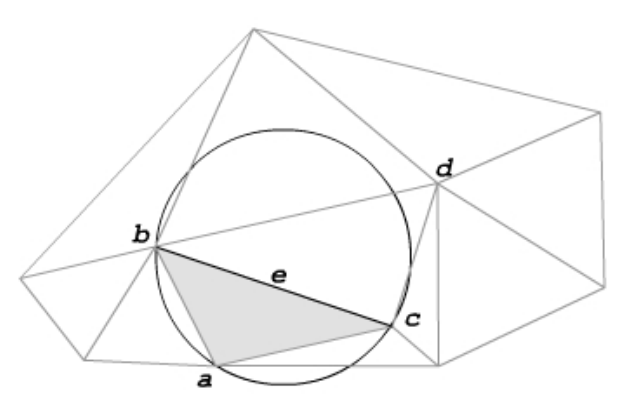

Figura 2.1: Representação de uma aresta $e$ localmente Delaunay.

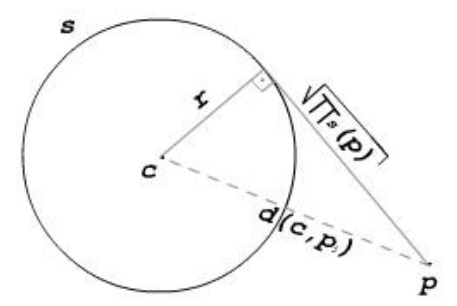

Figura 2.2: Representação geométrica da distância com peso.

Definição 2.1.9. A distância com peso, ou power distância, de um ponto p com relação a um círculo s é definida como:

$$
\Pi_{s}(p)=\|p-c\|^{2}-r^{2}
$$

onde c e r são o centro e o raio do círculo $s$, respectivamente.

Podemos notar que se $p$ está dentro de $s$ então $\Pi_{s}(p)<0$, se $p$ está sobre $s$ então $\Pi_{s}(p)=0$, se $p$ está fora de $s$ então $\Pi_{s}(p)>0$. Na figura 2.2, a distância com peso é interpretada geometricamente.

Teorema 2.1.1. Se todas as arestas de uma triangulação $K$ são localmente Delaunay então $K$ é triangulação de Delaunay.

Demonstração 2.1.1. Considere um triângulo abc e um vértice $v \in K$. Vamos mostrar que $v$ está fora do circuncírculo de abc.

Escolha um ponto $x$ no interior do triângulo abc tal que o segmento $x v$ não contenha outro vértice de $K$ além de $v$. Sejam os simplexos $\sigma_{0}, \sigma_{1}, \ldots, \sigma_{n}$ os simplexos interceptados pelo segmento $x v$, como ilustra a figura 2.3. Seja $\Pi_{i}(v)$ a distância com peso entre o vértice $v$ e o circuncentro do simplexo $\sigma_{i}$. 


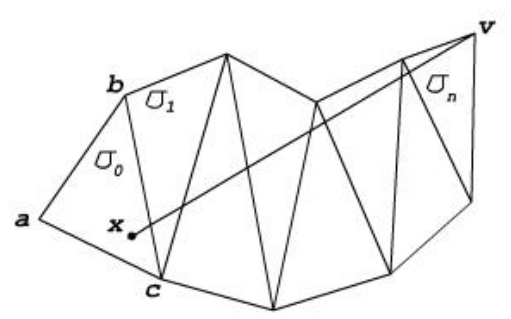

Figura 2.3: Seqüencia de simplexos interceptados pelo segmento $x v$.
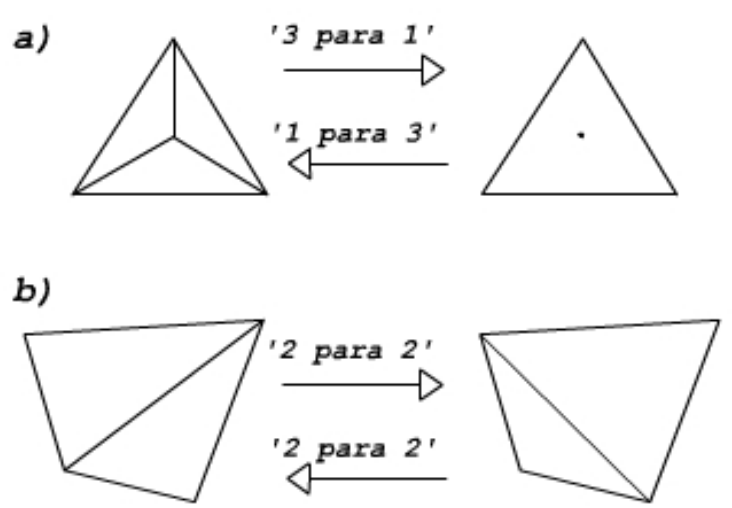

Figura 2.4: Possíveis flips em uma triangulação com 2 dimensões.

Como as arestas são localmente Delaunay temos $\Pi_{0}(v)>\Pi_{1}(v)>\ldots>\Pi_{n}(v)=0$ logo $\Pi_{0}(v)>0$ logo v não está contido no circuncírculo de abc.

Lema 2.1.1. Seja uma aresta e de uma triangulação K. Então e é localmente Delaunay ou é flipavel e a aresta gerada no flip é localmente Delaunay.

Uma aresta é flipavel se ela é diagonal de um quadrilátero convexo. Os flips em duas dimensões só podem ser de duas formas $2-2$ quando é trocada a diagonal de um quadrilátero ou 1 - 3 quando se insere um ponto em um triângulo, como ilustra a figura 2.4 .

A operação de flip em $\mathbb{R}^{2}$ pode ser definida como a projeção das faces inferiores de um tetraedro equilátero em $\mathbb{R}^{3}$ sobre $\mathbb{R}^{2}$. Tem-se os dois possíveis casos representados na figura 2.5:

Com o teorema 2.1.1 e o lema 2.1.1 pode-se desenvolver um algoritmo para calcular uma triangulação de Delaunay em 2D. Inicialmente constrói-se uma triangulação qualquer a partir dos pontos e para todos as arestas que não forem localmente Delaunay realizamos um flip $2-2$, assim todas as arestas serão localmente Delaunay e a triangulação será 


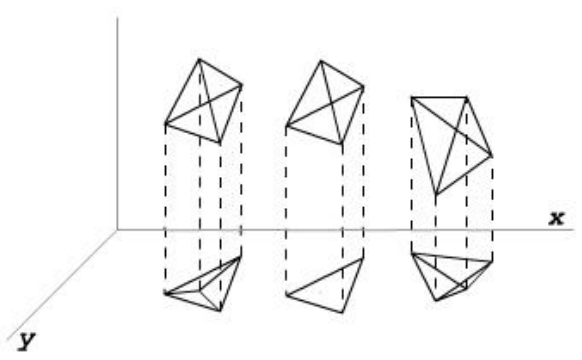

Figura 2.5: As duas primeiras projeções correspondem aos flips $1-3$ e $3-1$, quando se insere ou remove um ponto. A terceira projeção corresponde ao flip $2-2$.

Delaunay. Este algoritmo é conhecido como algoritmo de flip.

Outro algoritmo muito conhecido é o flip incremental, que utiliza também o teorema 2.1.1 como base. Neste algoritmo inicia-se com um triângulo com vértices no infinito que conterá todos os pontos que serão inseridos. Para cada ponto inserido procurase qual triângulo o contém, realiza-se um flip $1-3$ e enquanto existam triângulos não localmente Delaunay realiza-se flips $2-2$. A complexidade deste algoritmo é da ordem de $O\left(n^{2}\right)$, considerando uma triangulação com $n$ pontos, esta complexidade pode ser reduzida para $O(n \log n)$ com a utilização de estruturas de buscas adequadas. Mais detalhes deste algoritmo será dado no próximo capítulo.

Definição 2.1.10. Um ponto com peso é um ponto $p \in \mathbb{R}^{n}$ associado com um escalar $w_{p}^{2}$, $\hat{p}:\left(p, w_{p}^{2}\right) \in \mathbb{R}^{2} \times \mathbb{R}$. Graficamente um ponto com peso $\hat{p}$ é visto como um esfera de centro $p$ e raio $w_{p}$. A distância com peso entre dois pontos com peso é definido como:

$$
\Pi_{\hat{p}, \hat{q}}=\|p-q\|^{2}-w_{p}-w_{q}
$$

Dizemos que dois pontos com peso $\hat{p}$ e $\hat{q}$ são ortogonais se a distância com peso entre eles for zero, ou seja, $\hat{p}$ e $\hat{q}$ são ortogonais se $\Pi_{\hat{p}, \hat{q}}=0$. Dizemos que $\hat{p}$ e $\hat{q}$ são mais que ortogonais se $\Pi_{\hat{p}, \hat{q}} \geq 0$.

Definição 2.1.11. Sejam $\hat{p}, \hat{q}$ e $\hat{r} 3$ pontos com peso em $\mathbb{R}^{2} \times \mathbb{R}$, que formam um 2simplexo $\sigma$. Definimos como ortocírculo de $\sigma$ o círculo $\hat{z}$, ou ponto com peso, que satisfaça $\prod_{\hat{p}, \hat{z}}=\Pi_{\hat{q}, \hat{z}}=\prod_{\hat{r}, \hat{z}}=0$.

Definição 2.1.12. Uma aresta e de uma triangulação $K$ é dita localmente convexa se: 


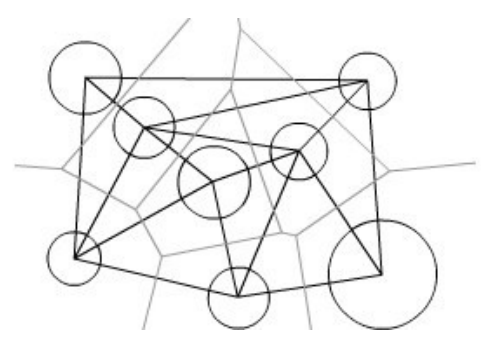

Figura 2.6: Ilustra um pequeno exemplo de triangulação Regular.

1. e pertence a apenas um triângulo e está no bordo do fecho convexo.

2. e pertence a dois triângulos abc e abd e o ponto com peso $\hat{d}$ está fora do ortocírculo $\hat{z}$ de abc, ou seja, $\Pi_{\hat{d}, \hat{z}} \geq 0$.

Definição 2.1.13. Dado um conjunto de pontos com peso em posição geral $P=\left\{\hat{p}_{0}, \ldots, \hat{p}_{m}\right\}$ $\in \mathbb{R}^{n}$, a triangulação regular, ilustrada na figura 2.6, de $P$ é um n-complexo simplicial $K$ satisfazendo:

1. A união dos simplexos em $K$ gera o fecho convexo de $P$.

2. A ortosfera de todos os n-simplexos é mais que ortogonal com relação aos pontos de $P$ (não considerando os vértices do simplexo), ou seja, todas as arestas são localmente convexas.

Na triangulação regular pode ocorrer que um ponto com peso sempre esteja contido no ortocírculo de algum triângulo, neste caso este ponto não pertencerá a nenhum triângulo e será chamado de ponto redundante.

O algoritmo para encontrar uma triangulação regular, ou Delaunay com peso, é o mesmo da triangulação de Delaunay sem peso mudando o teste de círculo vazio, pelo teste de ortocírculo mais que ortogonal. Pela possibilidade de existirem pontos redundantes poderão ocorrer flips $1-3$ ou $3-1$ sem a inserção ou remoção de pontos, como ilustra a figura 2.7 .

Em três dimensões a triangulação regular possui a propriedade de ortosfera mais que ortogonal, conseqüentemente é possível definir analogamente face localmente convexa e utilizarmos o algoritmo de flip incremental para a construção da triangulação regular. Neste caso, os flips podem ser $1-4$ ou $2-3$, representados pela figura 2.8. 


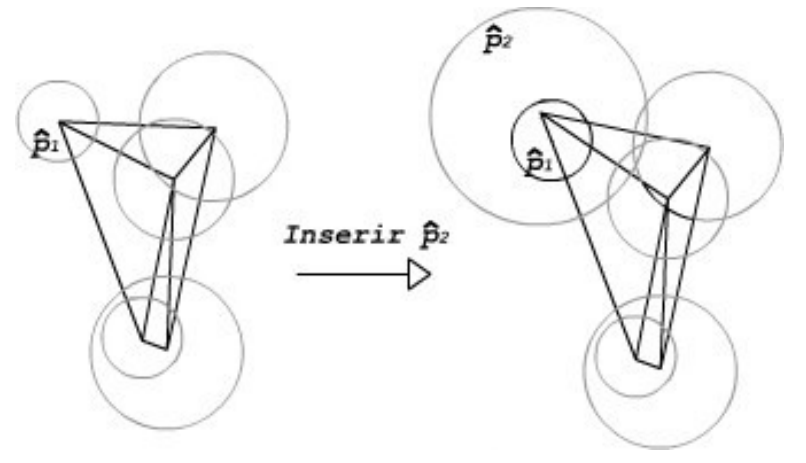

Figura 2.7: Exemplo onde ocorre um flip $3-1$ no ponto $\hat{p}_{1}$ ao inserir o ponto com peso $\hat{p}_{2}$.

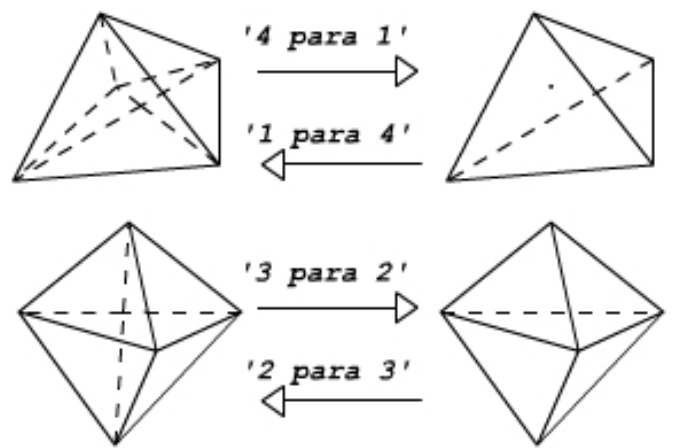

Figura 2.8: Possíveis flips em uma triangulação com 3 dimensões. 


\subsection{Triangulação Regular em Superfícies}

Quando o conjunto de pontos descreve uma superfície $\mathbb{R}^{3}$ alguns problemas surgem, tornando possível infinitas diferentes definições para triangulação regular.

O problema está relacionado com a definição de ortosfera, pois, utilizando da mesma definição para triangulações regulares em $\mathbb{R}^{3}$ existem infinitas esferas que passam pelos três pontos que definem um triângulo, desta forma é necessário escolher um destes pontos como centro para determinar a ortosfera, a seguir duas possíveis definições são apresentadas:

Definição 2.2.1. Sejam $\hat{p}, \hat{q}$ e $\hat{r}$ três pontos com peso em $\mathbb{R}^{3} \times \mathbb{R}$, que formam um 2simplexo $\sigma$. Definimos como ortosfera de $\sigma$ a esfera $\hat{z}$, ou ponto com peso, que satisfaça $\Pi_{\hat{p}, \hat{z}}=\Pi_{\hat{q}, \hat{z}}=\Pi_{\hat{r}, \hat{z}}=0$ e seu centro satisfaça pelo menos uma das condições abaixo:

1. O centro de $\hat{z}$ pertence a superfície.

2. O centro de $\hat{z}$ está contido no plano definido por $\hat{p}, \hat{q}$ e $\hat{r}$, ou seja, o ortocentro que define a ortosfera de menor raio.

A primeira definição, que exige que o ortocentro esteja contido na superfície [4], tem como vantagens:

1. O ortocentro é sempre um ponto contido na superfície, logo, podem ser adicionados como novos vértices à triangulação,em caso de refinamento de malha por exemplo.

2. É fácil determinar se um ponto está dentro ou fora da ortosfera, apenas calculando a distância em $\mathbb{R}^{3}$.

3. Determinar o ortocentro é basicamente equivalente a calcular a intersecção de uma reta com uma superfície, já que a união de todos os ortocentros possíveis descreve uma reta.

Como desvantagens, esta definição apresenta:

1. Não aplicável quando não existe função que descreve a superfície. 
2. A reta definida por todos os possíveis ortocentros pode ter como intersecção com a superfície vários pontos ou pode não existir.

A segunda definição, a qual exige que o ortocentro esteja contido no plano definido por $\hat{p}, \hat{q}$ e $\hat{r}$, possui as mesmas vantagens que a primeira, exceto pela possibilidade de adicionado como novo ponto, e é aplicável para qualquer triangulação.

Neste trabalho será utilizada a segunda definição, pois, as superfícies estudadas foram obtidas por meio de escaneamentos de objetos e não dispomos de funções que as descrevem.

Definição 2.2.2. Dado um conjunto de pontos com peso em posição geral $P=\left\{\hat{p}_{0}, \ldots, \hat{p}_{m}\right\}$ $\in \mathbb{R}^{3}$ que descrevam uma superfície, a triangulação regular de $P$ é um 2-complexo simplicial $K$ satisfazendo:

1. A união dos simplexos em $K$ é o bordo do fecho convexo de $P$.

2. A ortosfera de todos os n-simplexos é mais que ortogonal com relação aos pontos de $P$ (não considerando os vértices do simplexo), ou seja, todas as arestas são localmente convexas.

\subsection{Diagrama de Voronoi}

O diagrama de Voronoi com peso, ou power diagrama, de um conjunto de pontos com peso $P=\left\{\hat{p}_{0}, \hat{p}_{1}, \ldots, \hat{p}_{m}\right\}$ é o dual geométrico da triangulação regular, ou seja, para cada ponto da triangulação associa-se uma célula do diagrama cujos vértices são os ortocentros dos simplexos que contêm este ponto. As definições e propriedades aqui enunciadas foram baseadas em $[6,20]$.

Definição 2.3.1. Dado um conjunto de pontos $P=\left\{p_{0}, p_{1}, \ldots, p_{m}\right\} \in \mathbb{R}^{n}$, a região de Voronoi, ou polígono de Voronoi, associada ao sítio $p_{i}$ de $P$, representada por $V\left(p_{i}\right)$, é a região que contém todos os pontos mais próximos $p_{i}$ a qualquer outro ponto de $P$, ou seja,

$$
V\left(p_{i}\right)=\left\{x \in \mathbb{R}^{n} \mid d\left(p_{i}, x\right) \leq d\left(p_{j}, x\right) ; 0 \leq j \leq m\right\}
$$

onde d denota a distância Euclidiana em $\mathbb{R}^{n}$. 


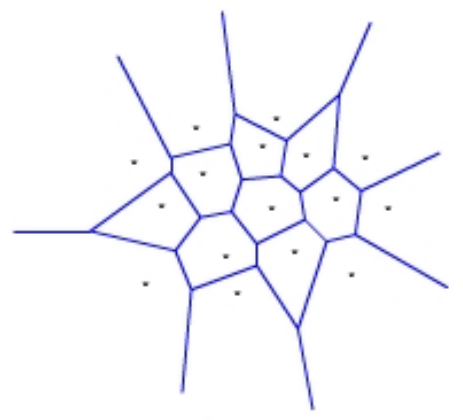

Figura 2.9: Todas as arestas são aresta de Voronoi do diagrama de Voronoi deste conjunto de pontos em $\mathbb{R}^{2}$, a união de cada três arestas é um vértice de Voronoi.

Definição 2.3.2. O conjunto $\operatorname{Vor}(P)=\left\{V\left(p_{0}\right), V\left(p_{1}\right), \ldots, V\left(p_{m}\right)\right\}$ é chamado de diagrama de Voronoi de $P$.

Em outras palavras, dado um conjunto de pontos $P=\left\{p_{0}, p_{1}, \ldots, p_{m}\right\} \in \mathbb{R}^{n}$, o diagrama de Voronoi é uma subdivisão espacial em regiões formadas pelos pontos mais próximos a cada um dos pontos de $P$, cada ponto de $P$ é conhecido como sítio. Chama-se de aresta de Voronoi o conjunto de pontos que pertencem simultaneamente a $n$ regiões de Voronoi, chama-se de vértice de Voronoi o ponto que pertence simultaneamente a $n+1$ regiões de Voronoi, ou seja, ponto eqüidistante a $n+1$ sítios, como ilustra 2.9.

Teorema 2.3.1. Se v é um vértice de Voronoi então v está na intersecção de $n+1$ arestas de Voronoi.

Teorema 2.3.2. Para cada vértice de Voronoi $v$, o interior do círculo $C(v)$ que passa por $n+1$ sítios cuja região de Voronoi contém $v$, e possui $v$ como centro não contém nenhum outro sítio em seu interior.

Demonstração 2.3.1. Supondo que exista um sítio $p_{i}$, onde $V\left(p_{i}\right)$ não contém $v$, contido no círculo de $C(v)$ então $d\left(p_{i}, v\right)<r$, onde $r$ é o raio de $C(v)$, que é absurdo pois os sítios que geraram $v$ distam $r$ de $v$ e o possuem em sua região de Voronoi.

Note que $C(v)$ a circunsfera de $n+1$ sítios, cujo centro é um vértice de Voronoi, e é vazia de pontos, este fato é a principal ligação entre diagrama de Voronoi e triangulações de Delaunay. 


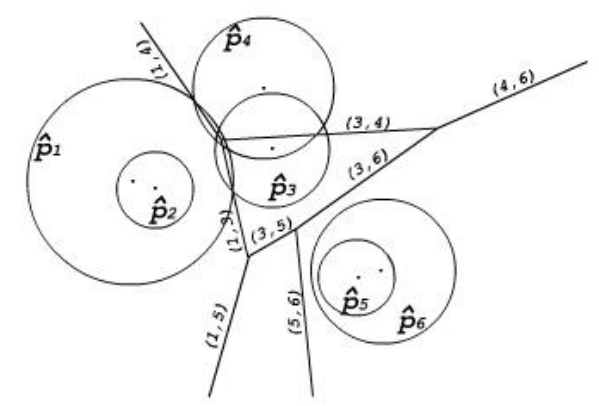

Figura 2.10: Simples exemplo de um power diagrama.

Teorema 2.3.3. A região de Voronoi $V\left(\right.$ pi) é limitada se e somente se $p_{i}$ pertence ao interior do fecho convexo de P.

Definição 2.3.3. Dado um conjunto de pontos com peso $P=\left\{\hat{p}_{0}, \hat{p}_{1}, \ldots, \hat{p}_{m}\right\} \in \mathbb{R}^{n}$ definimos a região de Voronoi associada ao sítio $p_{i}$ de $P$ como

$$
V\left(\hat{p}_{i}\right)=\left\{x \in \mathbb{R}^{n} \mid \Pi_{\hat{p}_{i}}(x) \leq \Pi_{\hat{p}_{j}}(x) ; 0 \leq j \leq m\right\}
$$

Definição 2.3.4. O conjunto $\operatorname{Vor}(P)=\left\{V\left(p_{0}\right), V\left(p_{1}\right), \ldots, V\left(p_{m}\right)\right\}$ é chamado de diagrama de Voronoi com peso de $P$, ou power diagrama de $P$.

A figura 2.10 ilustra um exemplo de diagrama de Voronoi com peso. 



\section{Capítulo 3}

\section{Algoritmos}

Neste capítulo estão descritos alguns resultados conhecidos que dão base para o desenvolvimento do trabalho proposto. Este capítulo divide-se em duas partes, na primeira são descritos trabalhos relacionados à triangulação de Delaunay algumas propriedades e algoritmos. Na segunda parte são descrito um algoritmo para calcular o power diagrama de um conjunto.

\subsection{Triangulação Regular}

Nesta seção descrevemos alguns tópicos relacionados com triangulações regulares, os quais serão de grande importância para o desenvolvimento do projeto. Além dos textos descritos existem muitos outros estudos relacionados com triangulações regulares, como exemplo [10], [11], [19], [1] e [15].

\subsubsection{Algoritmo Incremental com Flip}

Muitos dos algoritmos utilizados para a construção de uma triangulação regular são baseados em flip de arestas. Por outro lado, o algoritmo mais simples para construção da triangulação de Delaunay não funciona para triangulações regulares. Além disso, generalização deste algoritmo para três dimensões é complicada e foi demonstrado que não pode ser aplicada mesmo em triangulações de Delaunay.

Um exemplo onde o algoritmo pode falhar é dado pelo conhecido politopo de Schönhardt 


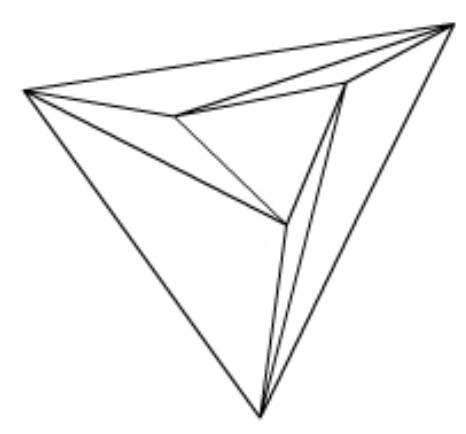

Figura 3.1: Politopo de Schönhardt, contra exemplo para o algoritmo de flip.

$\in \mathbb{R}^{2}$. Este politopo é o menor exemplo que não pode ser triangulado com o algoritmo de flip. Na figura 3.1, todas as arestas localmente convexas são flipáveis e todas as arestas não localmente convexas não são flipáveis.

Desta forma, Edelsbrunner e Shah [9] propuseram um algoritmo incremental para construção de uma triangulação regular para qualquer dimensão. Para a construção de uma triangulação regular de um conjunto de pontos com peso $P=\left\{\hat{p}_{0}, \hat{p}_{1}, \ldots, \hat{p}_{m}\right\} \in$ $\mathbb{R}^{2}$ incrementalmente é necessária uma triangulação inicial que contenha os pontos de $P$ que serão inseridos um a um. Desta forma, criam-se 3 pontos artificiais que serão convenientemente escolhidos com coordenadas no infinito, com seus pesos iguais a zero. Estes 3 pontos formam um único 2 - simplexo logo será uma triangulação regular.

Dada a triangulação inicial inserimos um a um os pontos de $P$. Para cada ponto inserido procura-se qual triângulo o contém. Caso este ponto não seja redundante, ele é inserido com um flip "1 para 3" e para todas as arestas que não sejam localmente convexas são realizados flips "2 para 2" ou "3 para 1"tornando a triangulação novamente regular. Este processo é repetido para todos os pontos de $P$. Com a remoção dos pontos artificiais (vértices no infinito) e todos os simplexos que os possuem como vértice obtém-se a triangulação regular de $P$. Abaixo é apresentado um pseudo-código para este algoritmo:

1 Construir uma triangulação inicial $T$ com vértices no infinito

2 Para cada ponto $p_{i}$ de $P$ faça:

3 Procure pelo triângulo $\sigma$ de $T$ que contém $p_{i}$

4 Se este $p_{i}$ não for redundante faça: 
$5 \quad \operatorname{Flip} 1-3\left(p_{i}, \sigma\right)$

6 Enquanto existirem simplexos não localmente convexos faça:

$7 \quad$ Procure a face $e$ não regular que seja flipável

$8 \quad$ Flip $(e) / /$ podem ocorrer flips 2-2 e flips 3-1

9 Remova os pontos artificiais e seus simplexos.

Note que na linha sete do pseudo-código tomamos cuidado para não pegar uma face que seja não localmente convexa e não flipável, porém existirão outras faces não localmente convexas que após flipadas tornarão esta face flipável.

Sabe-se que um $d$-simplexo é formado por $d+1$ pontos, logo n pontos formam $\left(\begin{array}{l}n \\ d+1\end{array}\right)$ $d$-simplexos. Isto implica que o número máximo de flips necessários para construir a triangulação regular é no máximo $2\left(\begin{array}{l}n \\ d+1\end{array}\right)=O\left(n^{(d+1) / 2}\right)$. A prova da corretude do algoritmo pode ser encontrada em [9].

\subsubsection{Triangulações Dinâmicas}

Nesta seção é apresentada uma estrutura de dados simples que permite um trabalho dinâmico, isto é, quando alguma alteração é feita no peso de algum ponto removemos este ponto da malha e o re-inserimos com o novo peso. Esta estrutura foi proposta em [22].

Existem vários métodos para remoção de pontos em uma triangulação de Delaunay, porém, para triangulações regulares o problema é mais complicado pois pontos redundantes podem voltar a pertencer à triangulação. Desta forma, é preciso uma estrutura que possa armazenar todos os pontos, redundantes ou não, para facilitar a operação de remoção. Neste caso, utilizaremos uma estrutura onde para cada ponto serão armazenados suas coordenadas e um rótulo, que será usado na remoção. Cada n-simplexo possui ponteiros para seus vértices e ponteiros para seus vizinhos. Assim, como o algoritmo incremental com flip, os pontos serão inseridos em uma triangulação já existente. Criam-se vértices artificiais cujo simplexo formado por eles conterá todos os pontos do conjunto que estamos trabalhando. Estes vértices terão coordenadas no infinito e pesos zero, exceto por 
um que terá peso infinito. Este ponto com peso infinito é a chave para o funcionamento nos algoritmos de inserção e remoção.

No caso 3D quando um ponto $p$ é inserido procura-se qual o tetraedro que o contém, por exemplo $p_{a}, p_{b}, p_{c}$ e $p_{d}$ com rótulos $a, b, c$ e $d$ respectivamente. Assim o ponto $p$ é inserido com um flip $1-4$ e as novas faces criadas são inseridas em uma lista. Enquanto esta lista não estiver vazia será removida uma face e caso seja necessário, esta face será flipada. Para cada face flipada, as novas faces criadas serão inseridas na lista. Quando não for mais necessário realizar flips marcamos o rótulo de $p$ como $\max (a, b, c, d)$, se $\max (a, b, c, d)=\infty$ atribuímos o rótulo de $p$ como $N+1$, onde $N$ indica o número de inserções realizadas.

Para remover um ponto $p$ realizamos flips em todas os simplexos contendo $p$ até que ele pertença a apenas 4 simplexos, assim é possível realizar um flip $4-1$ e o ponto $p$ será removido. Alterando o peso conforme desejado, $p$ é re-inserido na triangulação. Cada ponto redundante contido nos triângulos originais de $p$ devem ser verificados se retornarão à triangulação.

\subsubsection{Hiperplano Associado}

Hiperplano: Para cada ponto com peso $\hat{p} \in \mathbb{R}^{3}$ seja $\eta_{p}(x): \mathbb{R}^{3} \rightarrow \mathbb{R}$ a função definida por $\eta_{p}(x)=\|x\|^{2}-\Pi_{p}(x)=2<x, p>-\|p\|^{2}+w_{p}$, cujo gráfico é um hiperplano do $\mathbb{R}^{4}$ (ou um plano em $\mathbb{R}^{3}$ se $\hat{p} \in \mathbb{R}^{2}$ ). Note que se $w_{p}=0$ então o plano definido por $\eta_{p}$ é tangente ao parabolóide $\|x\|^{2}$. De forma mais geral se $w_{p}>0, \eta_{p}$ intercepta o parabolóide $\|x\|^{2}$, como ilustra a figura 3.2.

Se os planos para todos os pontos com peso $P \in \mathbb{R}^{2}$ forem calculados, pode-se mostrar [7] que a projeção do envelope superior dos pontos sobre $\mathbb{R}^{2}$ fornece o power diagrama de $P$. Isso decorre do fato que a intersecção de três planos, gerados por três pontos com peso, quando projetado em $\mathbb{R}^{2}$ dá origem ao ortocentro destes três pontos com peso. Quando um plano não está no envelope superior significa que este ponto não possui uma região de Voronoi, ou seja, ele é redundante.

Como o power diagrama é o dual geométrico da triangulação regular é possível utiliza- 


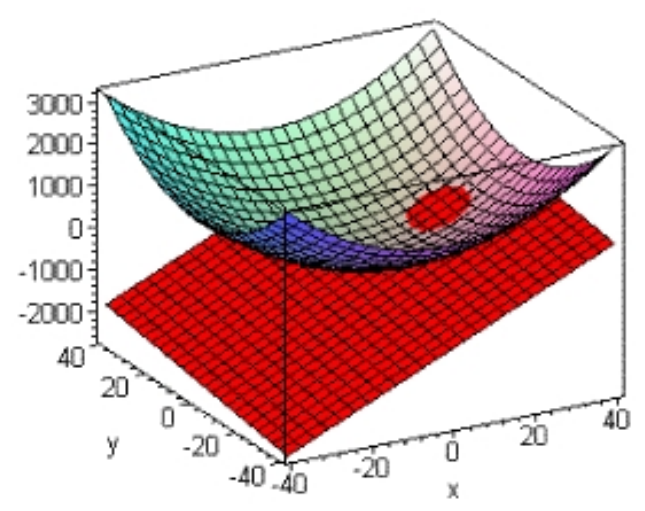

Figura 3.2: Neste exemplo temos $\hat{p} \in \mathbb{R}^{2}$ cujo plano $\eta_{p}$ se intercepta no parabolóide $\|x\|^{2}$.

lo para encontrar a triangulação regular de um conjunto de pontos com peso. A visão da triangulação do ponto de vista dos planos associados aos pontos com peso permite entender melhor a dinâmica da triangulação frente à mudança dos pesos. De fato, quando se aumenta o peso de um ponto, seu plano irá subir. Se o peso do ponto diminuir, o plano irá descer. Enquanto à intersecção entre os planos não afete a relação de incidência e adjacência das faces do envelope superior, nenhum flip irá ocorrer. Caso a mudança nos pesos afete as intersecções entre os planos de modo a modificar as incidências das faces do envelope superior, flips irão ocorrer na triangulação 2D. A figura 3.3 ilustra este processo de alteração de peso.

\subsection{Diagrama de Voronoi com Peso}

Uma outra forma para encontrar a triangulação regular de um conjunto é determinar o diagrama de Voronoi com peso do conjunto e a partir dele construir a triangulação regular, onde, para cada vértice de Voronoi é atribuído um único triângulo cujos vértices são os pontos que têm como ortocentro este vértice de Voronoi. Nesta seção descrevemos um algoritmo que determina o diagrama de Voronoi e o estendemos para diagrama de Voronoi com peso.

Além deste algoritmo, outros algoritmos relacionados com triangulações regulares $[23,3]$ e propriedades $[2,8]$ podem ser encontradas na literatura (estes algoritmos não constroem triangulações regulares diretamente). 

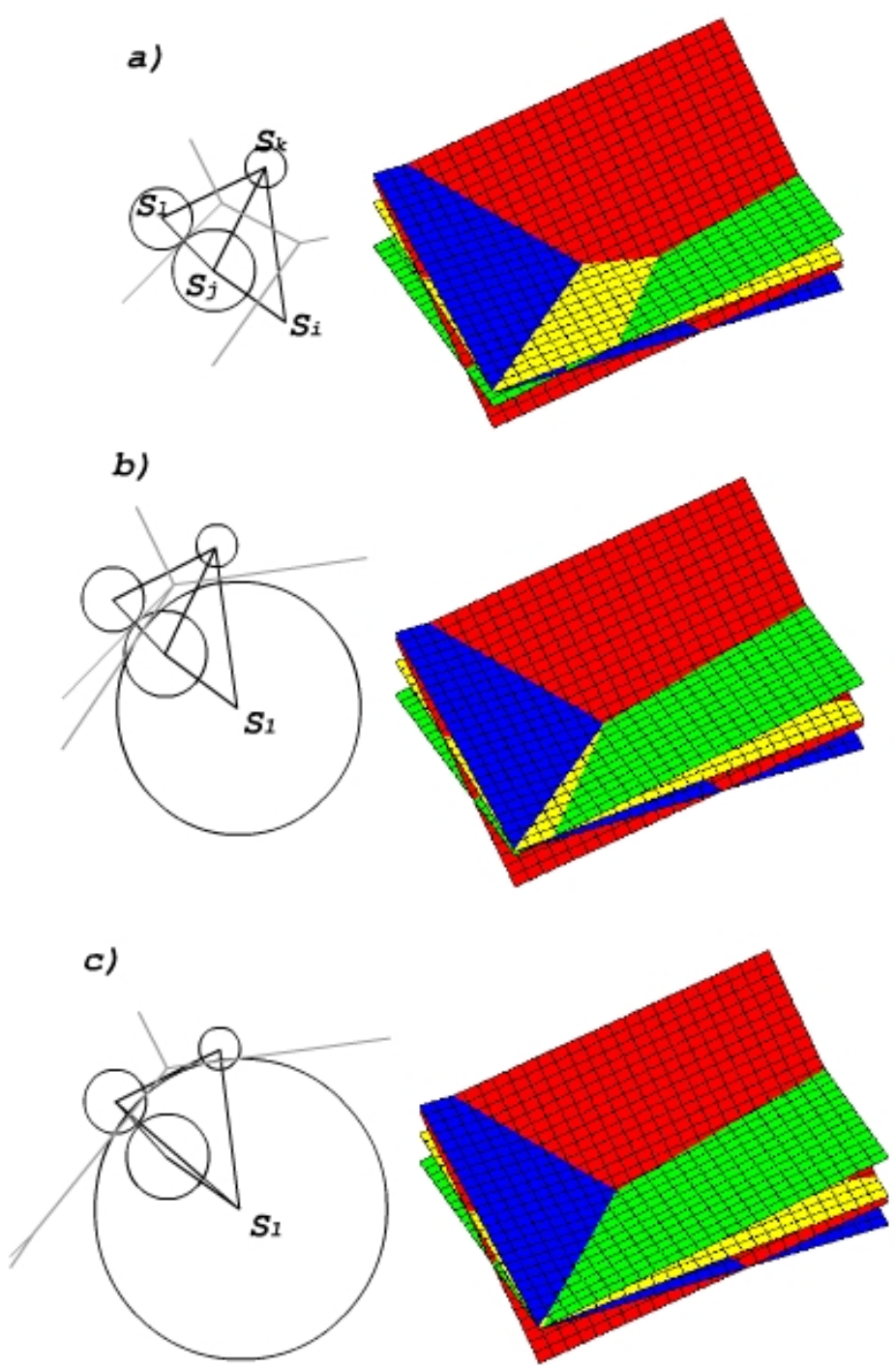

Figura 3.3: Neste exemplo o ponto $s_{i}$ tem seu peso alterado. Enquanto não há alteração na relação de incidência entre as faces do envelope superior, nenhum flip é realizado na triangulação, como mostra a figura a) e b). A mudança nas faces do envelope superior indica um flip de arestas na triangulação, como ilustra a figura c). 


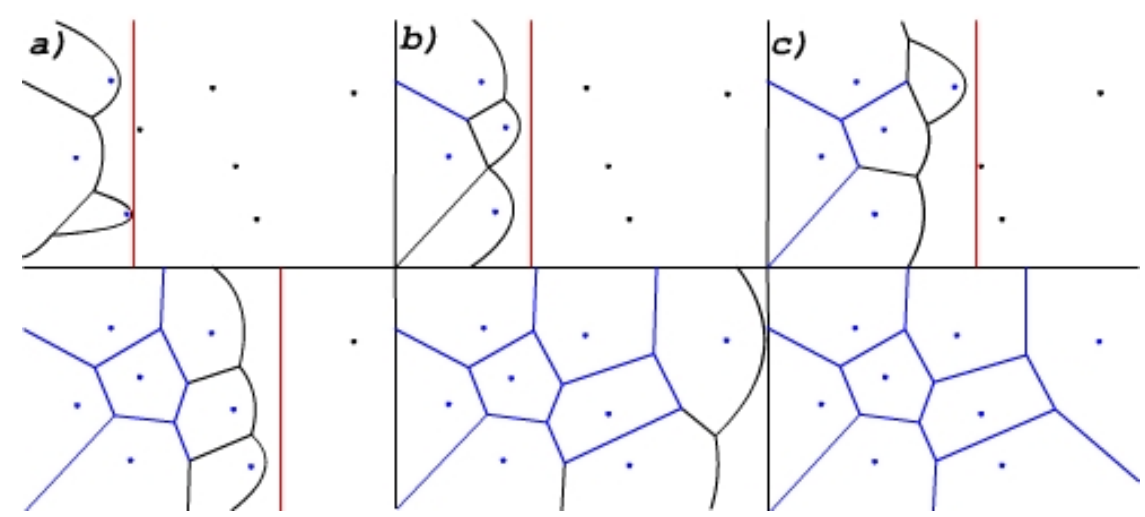

Figura 3.4: Inicialmente temos uma reta vertical passando pelos pontos, criando as respectivas parábolas. Na imagem a) houve apenas intersecções entre duas parábolas criando segmentos de retas, na imagem b) houve intersecção entre as três parábolas entre os três pontos azuis superiores criando um vértice de Voronoi. Nas demais imagens a reta continua movendo até que todos os vértices estejam a sua esquerda e não haja mais intersecções entre 3 parábolas.

\subsubsection{Algoritmo Sweepline}

Um algoritmo bem conhecido para encontrar o diagrama de Voronoi de um conjunto é o algoritmo desenvolvido por Fortune [13], que pode ser estendido para a construção do diagrama de Voronoi com peso de um conjunto. Este algoritmo pode ter complexidade $O(n \log n)$ quando acompanhado por estruturas adequadas.

Em duas dimensões, o algoritmo funciona com uma reta $r$, conhecida como sweepline, caminhando, da esquerda para direita por exemplo, e para cada vértice ficar à esquerda de $r$ uma parábola entre $r$ e o vértice será definida. Desta forma, cada vértice à direita de $r$ terá uma parábola associada que varia conforme a reta $r$ caminha e, enquanto esta parábola varia, os pontos de intersecção entre varias parábolas também variam. A união de todos os pontos pertencente à intersecção de duas parábolas será a aresta de Voronoi dos respectivos vértices, caso o ponto seja intersecção de três parábolas este ponto será um vértice de Voronoi.

Quando uma parábola estiver totalmente contida à esquerda da curva formada pela união das demais parábolas, esta não será mais considerada na construção do diagrama de Voronoi, pois já terá toda sua região de Voronoi encontrada. A imagem 3.4 ilustra alguns passos desde algoritmo para facilitar a compreensão de seu funcionamento.

Este algoritmo pode ser estendido para três dimensões substituindo as parábolas por 
parabolóides e a reta $r$ por um plano $\eta$.

Para estender este algoritmo para construção do diagrama de Voronoi com peso de um conjunto de pontos é preciso substituir a parábola pela curva $s$ cuja distância de cada ponto de $s$ a reta $r$ e a distância com peso do mesmo ponto de $s$ ao vértice com peso sejam iguais, ou seja, $s=\left\{x \in \mathbb{R} \mid d(x, r)=\Pi_{p}(x)\right\}$ onde $p$ é um ponto com peso e $d$ denota a distância euclidiana.

Demonstração 3.2.1. Para demonstrar que este algoritmo funciona, basta mostrar que a intersecção de duas curvas s, como definida acima, será um ponto que tenha a mesma distância com peso aos pontos que geraram esta parábola.

Sejam dois pontos com peso $\hat{p}_{i}$ e $\hat{p}_{j}$ e uma reta $r$.

Se $x$ é um ponto da parábola gerada por $\hat{p}_{i}$ e $r$, temos, por definição, $d(x, r)=\Pi_{p_{i}}(x)$.

Se $x$ é um ponto da parábola gerada por $\hat{p}_{j}$ e r, temos, por definição, $d(x, r)=\Pi_{p_{j}}(x)$.

Se $x$ é ponto de intersecção destas duas parábolas, então $\Pi_{p_{i}}(x)=d(x, r)=\Pi_{p_{j}}(x)$, ou seja, $\Pi_{p_{i}}(x)=\Pi_{p_{j}}(x)$, logo $x$ é ponto da aresta de Voronoi de $\hat{p}_{i}$ e $\hat{p}_{j}$. 


\section{Capítulo 4}

\section{Desenvolvimentos Realizados}

Neste capítulo estão descritos os trabalhos realizados mostrando todos os passos que levaram ao desenvolvimento de um algoritmo para alterar pesos de uma triangulação sem que seja necessário remover o ponto da triangulação, ao contrário do algoritmo proposto por Marc Vigo et al.[22].

Partindo da idéia de usar flips para alteração de pesos, será descrita a técnica que foi desenvolvida para resolver o problema de encontrar os pesos para os vértices de modo a tornar uma triangulação qualquer em triangulação regular, desde que a triangulação inicial seja convexa. Em termos matemáticos, busca-se resolver o seguinte problema: dado um conjunto finito de pontos $P=\left\{p_{1}, p_{2}, p_{3}, \ldots, p_{n}\right\}$, que constituem os vértices de uma triangulação $T$, é necessário determinar o peso de cada ponto $p_{i}$ que torne a triangulação $T$ uma triangulação regular.

Pode-se notar que tal distribuição de pesos não é única, podendo nem mesmo existir para uma determinada triangulação $T$, pois não existe nenhuma garantia teórica sobre este problema, porém mostramos que ,se existe solução, nossa técnica irá encontrá-la.

\subsection{Prevendo um flip}

Como descrito anteriormente, flips podem ser usados para construir uma triangulação regular. Utilizando este fato, procuramos uma forma para prever quando um flip irá ocorrer ao se alterar o peso de um ponto. 
Para prever quando um flip irá ocorrer associamos a cada vértice um plano em $\mathbb{R}^{3}$ como descrito anteriormente. Sabe-se que a intersecção de três planos é um ponto e a projeção vertical deste ponto é o ortocentro do ortocírculo dos três vértices correspondentes.

Demonstração 4.1.1. Para demonstrar que a projeção vertical da intersecção de três planos é o ortocentro dos respectivos pontos que geraram estes planos, precisaremos conhecer as coordenadas do ortocentro. Sejam os pontos com peso $p_{i}=\left(x_{i}, y_{i}, w_{i}\right), p_{j}=$ $\left(x_{j}, y_{j}, w_{j}\right)$ e $p_{k}=\left(x_{k}, y_{k}, w_{k}\right)$ que forma um triângulo $p_{i} p_{j} p_{k}$. Podemos encontrar o ortocentro deste triângulo resolvendo o seguinte sistema linear:

$$
\left\{\begin{array}{l}
\Pi_{p_{i}}(z)=\Pi_{p_{j}}(z) \\
\Pi_{p_{i}}(z)=\Pi_{p_{k}}(z)
\end{array}\right.
$$

onde $z=\left(x_{z}, y_{z}\right)$ é o ortocentro do triângulo $p_{i} p_{j} p_{k}$ ou seja:

$$
\left\{\begin{array}{l}
2\left(x_{j}-x_{i}\right) x_{1}+2\left(y_{j}-y_{i}\right) y_{1}=x_{j}{ }^{2}-x_{i}{ }^{2}+y_{j}{ }^{2}-y_{i}{ }^{2}+w_{i}{ }^{2}-w_{j}{ }^{2} \\
2\left(x_{k}-x_{i}\right) x_{1}+2\left(y_{k}-y_{i}\right) y_{1}=x_{k}{ }^{2}-x_{i}{ }^{2}+y_{k}{ }^{2}-y_{i}{ }^{2}+w_{i}{ }^{2}-w_{k}{ }^{2}
\end{array}\right.
$$

Resolvendo esse sistema pelo método de Crammer temos:

$$
\begin{aligned}
& x_{z}=\frac{B_{1}\left(C_{2}+w_{i}\right)-B_{2}\left(C_{1}+w_{i}\right)}{A_{1} B_{2}-A_{2} B_{1}} \\
& y_{z}=\frac{A_{2}\left(C_{1}+w_{i}\right)-A_{1}\left(C_{2}+w_{i}\right)}{A_{1} B_{2}-A_{2} B_{1}}
\end{aligned}
$$

onde: $A_{1}=2\left(x_{i}-x_{j}\right), A_{2}=2\left(x_{i}-x_{k}\right), B_{1}=2\left(y_{i}-y_{j}\right), B_{2}=2\left(y_{i}-y_{k}\right), C_{1}=$ $\left\|p_{j}\right\|^{2}-\left\|p_{i}\right\|^{2}-w_{j}$ e $C_{2}=\left\|p_{k}\right\|^{2}-\left\|p_{i}\right\|^{2}-w_{k}$.

Com o ortocentro determinado, podemos continuar a demonstração inicial, associando a cada ponto com peso $\hat{p}_{i}, \hat{p}_{j}$ e $\hat{p}_{k}$ temos:

$$
\begin{aligned}
& \eta_{p_{i}}(x)=2<(x, y),\left(x_{i}, y_{i}\right)>-\left\|p_{i}\right\|^{2}+w_{i} \\
& \eta_{p_{j}}(x)=2<(x, y),\left(x_{j}, y_{j}\right)>-\left\|p_{j}\right\|^{2}+w_{j} \\
& \eta_{p_{k}}(x)=2<(x, y),\left(x_{i}, y_{k}\right)>-\left\|p_{k}\right\|^{2}+w_{k}
\end{aligned}
$$

Calculando a diferença entre $\eta_{p_{i}}(x)-\eta_{p_{j}}(x)$ temos: 


$$
\eta_{p_{i}}(x)-\eta_{p_{j}}(x)=2\left(x\left(x_{i}-x_{j}\right)\right)+y\left(y_{i}-y_{j}\right)+w_{i}-w_{j}+\left\|p_{j}\right\|^{2}-\left\|p_{i}\right\|^{2}
$$

Utilizando a mesma substituição de variáveis acima temos:

$$
\eta_{p_{i}}(x)-\eta_{p_{j}}(x)=x A_{1}+y B_{1}+C_{1}+w_{i}
$$

Fazendo $(x, y)=\left(x_{z}, y_{z}\right)$, isto é, nas coordenadas do ortocentro de $p_{i} p_{j} p_{k}$, em $\eta_{p_{i}}(x)-$ $\eta_{p_{j}}(x)$ temos:

$\eta_{p_{i}}(x)-\eta_{p_{j}}(x)=\frac{A_{1}\left(B_{1}\left(C_{2}+w_{i}\right)-B_{2}\left(C_{1}+w_{i}\right)\right)}{A_{1} B_{2}-A_{2} B_{1}}+\frac{B_{1}\left(A_{2}\left(C_{1}+w_{i}\right)-A_{1}\left(C_{2}+w_{i}\right)\right)}{A_{1} B_{2}-A_{2} B_{1}}+C_{1}+w_{i}$

$$
\eta_{p_{i}}(x)-\eta_{p_{j}}(x)=\frac{A_{1} B_{1}\left(C_{2}+w_{i}-\left(C_{2}+w_{i}\right)\right)-\left(A_{1} B_{2}-A_{2} B_{1}\right)\left(C_{1}+w_{i}\right)}{A_{1} B_{2}-A_{2} B_{1}}+C_{1}+w_{i}
$$

$$
\eta_{p_{i}}(x)-\eta_{p_{j}}(x)=0-\left(C_{1}+w_{i}\right)+C_{1}+w_{i}=0
$$

Logo temos que $\eta_{p_{i}}(x)=\eta_{p_{j}}(x)$ em $(x, y)=\left(x_{z}, y_{z}\right)$. Agora calculando a diferença $\eta_{p_{i}}(x)-\eta_{p_{k}}(x)$ em $(x, y)=\left(x_{z}, y_{z}\right)$ temos:

$$
\begin{gathered}
\eta_{p_{i}}(x)-\eta_{p_{k}}(x)=2\left(x\left(x_{i}-x_{k}\right)\right)+y\left(y_{i}-y_{k}\right)+w_{i}-w_{k}+\left\|p_{k}\right\|^{2}-\left\|p_{i}\right\|^{2} \\
\eta_{p_{i}}(x)-\eta_{p_{k}}(x)=x A_{2}+y B_{2}+C_{2}+w_{i}
\end{gathered}
$$

Fazendo $(x, y)=\left(x_{z}, y_{z}\right)$ em $\eta_{p_{i}}(x)-\eta_{p_{k}}(x)$ temos: 
$\eta_{p_{i}}(x)-\eta_{p_{k}}(x)=\frac{A_{2}\left(B_{1}\left(C_{2}+w_{i}\right)-B_{2}\left(C_{1}+w_{i}\right)\right)}{A_{1} B_{2}-A_{2} B_{1}}+\frac{B_{2}\left(A_{2}\left(C_{1}+w_{i}\right)-A_{1}\left(C_{2}+w_{i}\right)\right)}{A_{1} B_{2}-A_{2} B_{1}}+C_{2}+w_{i}$

$$
\eta_{p_{i}}(x)-\eta_{p_{k}}(x)=\frac{A_{2} B_{2}\left(C_{1}+w_{i}-\left(C_{1}+w_{i}\right)\right)-\left(A_{1} B_{2}-A_{2} B_{1}\right)\left(C_{2}+w_{i}\right)}{A_{1} B_{2}-A_{2} B_{1}}+C_{1}+w_{i}
$$

$$
\eta_{p_{i}}(x)-\eta_{p_{k}}(x)=0-\left(C_{2}+w_{i}\right)+C_{2}+w_{i}=0
$$

Logo temos que: $\eta_{p_{i}}(x)=\eta_{p_{j}}(x)=\eta_{p_{k}}(x)$ em $(x, y)=\left(x_{z}, y_{z}\right)$, ou seja, os três planos têm como intersecção o ponto $\left(x_{z}, y_{z}, \eta_{p_{i}}\left(x_{z}, y_{z}\right)\right)$, logo a projeção da intersecção dos três planos é o ortocentro do triângulo.

Sabe-se também que, quando dois ortocírculos de triângulos vizinhos têm o mesmo centro, situação singular onde quatro pontos são ortocirculares, um flip irá ocorrer entre os dois triângulos correspondentes.

Baseado neste dois fatos, um flip irá ocorrer quando quatro planos tiverem como intersecção um único ponto, ou seja, dois ortocentros serão os mesmos, e isto irá ocorrer quando os pesos de quatro pontos satisfizerem o seguinte sistema linear:

$$
\left\{\begin{array} { l } 
{ \eta _ { \hat { p } _ { i } } - \eta _ { \hat { p } _ { j } } = 0 } \\
{ \eta _ { \hat { p } _ { i } } - \eta _ { \hat { p } _ { k } } = 0 } \\
{ \eta _ { \hat { p } _ { i } } - \eta _ { \hat { p } _ { l } } = 0 }
\end{array} \Rightarrow \left\{\begin{array}{l}
-\left\|p_{i}\right\|^{2}+\left\|p_{j}\right\|^{2}+2\left\langle p_{i}, x\right\rangle-2\left\langle p_{j}, x\right\rangle+w_{p_{i}}-w_{p_{j}}=0 \\
-\left\|p_{i}\right\|^{2}+\left\|p_{k}\right\|^{2}+2\left\langle p_{i}, x\right\rangle-2\left\langle p_{k}, x\right\rangle+w_{p_{i}}-w_{p_{k}}=0 \\
-\left\|p_{i}\right\|^{2}+\left\|p_{l}\right\|^{2}+2\left\langle p_{i}, x\right\rangle-2\left\langle p_{l}, x\right\rangle+w_{p_{i}}-w_{p_{l}}=0
\end{array}\right.\right.
$$

Na forma matricial: 


$$
\left(\begin{array}{cccccc}
A_{1} & B_{1} & 1 & -1 & +0 & +0 \\
A_{2} & B_{2} & 1 & +0 & -1 & +0 \\
A_{3} & B_{3} & 1 & +0 & +0 & -1
\end{array}\right)\left(\begin{array}{c}
x \\
y \\
w_{p_{i}} \\
w_{p_{j}} \\
w_{p_{k}} \\
w_{p_{l}}
\end{array}\right)=\left(\begin{array}{c}
C_{1} \\
C_{2} \\
C_{3}
\end{array}\right)
$$

onde:

$$
\begin{array}{lll}
A_{1}=\left(x_{i}-x_{j}\right) & B_{1}=2\left(y_{i}-y_{j}\right) & C_{1}=\left\|p_{j}\right\|^{2}-\left\|p_{i}\right\|^{2} \\
A_{2}=\left(x_{i}-x_{k}\right) & B_{2}=2\left(y_{i}-y_{k}\right) & C_{2}=\left\|p_{k}\right\|^{2}-\left\|p_{i}\right\|^{2} \\
A_{3}=\left(x_{i}-x_{l}\right) & B_{3}=2\left(y_{i}-y_{l}\right) & C_{3}=\left\|p_{l}\right\|^{2}-\left\|p_{i}\right\|^{2}
\end{array}
$$

sendo $x_{i}, x_{j}, x_{k}$ e $x_{l} y_{i}, y_{j}, y_{k}$ e $y_{l}$ as coordenadas dos pontos $p_{i} p_{j} p_{k}$ e $p_{l}$ respectivamente.

Escalonando o sistema obtemos:

$$
\left(\begin{array}{llllll}
A_{1} & B_{1} & 1 & -1 & +0 & +0 \\
0 & \frac{A_{1} B_{2}-A_{2} B_{1}}{A_{1}} & \frac{A_{1}-A_{2}}{A_{1}} & \frac{A_{2}-A_{1}}{A_{1}} & -1 & +0 \\
0 & 0 & D_{1} & -D_{1} & \frac{A_{1} B_{3}-A_{3} B_{1}}{A_{1} B_{2}-A_{2} B_{1}}-1 & -1
\end{array}\right)\left(\begin{array}{c}
x \\
y \\
w_{p_{i}} \\
w_{p_{j}} \\
w_{p_{k}} \\
w_{p_{l}}
\end{array}\right)=\left(\begin{array}{c}
C_{1} \\
\frac{C_{2} A_{1}-C_{1} A_{2}}{A_{1}} \\
D_{2}
\end{array}\right)
$$

onde:

$$
\begin{gathered}
D_{1}=\frac{\left(A_{1}-A_{3}\right)\left(A_{1} B_{2}-A_{2} B_{1}\right)-\left(A_{1}-A_{2}\right)\left(A_{1} B_{3}-A_{3} B_{1}\right)}{A_{1}\left(A_{1} B_{2}-A_{2} B_{1}\right)} \\
D_{2}=\frac{\left(A_{1} C_{3}-A_{3} C_{1}\right)\left(A_{1} B_{2}-A_{2} B_{1}\right)-\left(A_{1} C_{2}-A_{2} C_{1}\right)\left(A_{1} C_{3}-A_{3} C_{1}\right)}{A_{1}\left(A_{1} B_{2}-A_{2} B_{1}\right)}
\end{gathered}
$$

Da última linha da matriz é possível determinar uma equação que em função dos pesos pode-se determinar quando os ortocírculos dos dois triângulos serão coincidentes. Após algumas manipulações a equação pode ser escrita como: 


$$
H w_{p_{i}}+w_{p_{j}}\left(A_{2} B_{3}-A_{3} B_{2}\right)+w_{p_{k}}\left(A_{3} B_{1}-A_{1} B_{3}\right)+w_{p_{l}}\left(A_{1} B_{2}-A_{2} B_{1}\right)=-G,
$$

onde:

$$
\begin{aligned}
& H=A_{1}\left(B_{2}-B_{3}\right)+A_{2}\left(B_{3}-B_{1}\right)+A_{3}\left(B_{1}-B_{2}\right) \\
& G=A_{1}\left(B_{2} C_{3}-B_{3} C_{2}\right)+A_{2}\left(B_{3} C_{1}-B_{1} C_{3}\right)+A_{3}\left(B_{1} C_{2}-B_{2} C_{1}\right)
\end{aligned}
$$

A equação 4.16 diz quando os quatro pontos se tornaram ortocirculares, assim é possível definir um limitante que nos garantirá a existência de alguma aresta, por exemplo, se os pesos satisfizerem:

$$
H w_{p_{i}}+w_{p_{j}}\left(A_{2} B_{3}-A_{3} B_{2}\right)+w_{p_{k}}\left(A_{3} B_{1}-A_{1} B_{3}\right)+w_{p_{l}}\left(A_{1} B_{2}-A_{2} B_{1}\right)>-G,
$$

estará garantido que a aresta formada pelos vértices $p_{i}$ e $p_{l}$ estarão na triangulação. Isto significa que, enquanto os pesos satisfizerem a equação 4.17, será possível alterar o peso de qualquer ponto sem que ocorra nenhuma alteração topológica; caso a alteração de pesos não satisfaça a equação 4.17 ocorrerá um flip e a aresta formada pelos vértices $p_{i}$ e $p_{l}$ não será uma aresta da triangulação regular.

$\mathrm{Na}$ verdade, outra equação pode ser desenvolvida a partir de 4.16:

$$
H w_{p_{i}}+w_{p_{j}}\left(A_{2} B_{3}-A_{3} B_{2}\right)+w_{p_{k}}\left(A_{3} B_{1}-A_{1} B_{3}\right)+w_{p_{l}}\left(A_{1} B_{2}-A_{2} B_{1}\right)<-G,
$$

e não é conhecido qual das duas equações 4.17 e 4.18 irá preservar a aresta $p_{i} p_{l}$. Para determinar qual equação usar, um simples teste é necessário: se $p_{i} p_{l}$ é uma aresta localmente Delaunay então a equação 4.17 será usada se $-G$ for negativo e 4.18 se $-G$ for positivo, pois desta forma as equações serão satisfeitas quando os pesos forem zero. Caso $p_{i} p_{l}$ não seja aresta localmente Delaunay 4.17 será usada se $-G$ for positivo e 4.18 se $-G$ for negativo. 


\subsection{Algoritmo para Triangulações Dinâmicas}

Partindo da equação 4.16 é possível construir um algoritmo para alterar pesos em uma triangulação regular sem precisar remover pontos, vamos supor que o peso $w_{p_{i}}$ do ponto $p_{i}$ terá seu peso alterado para $w_{p_{i}}+\epsilon$ assim teremos dois casos $\epsilon>0$ e $\epsilon<0$. Em ambos os casos será necessário procurar duplas de simplexos e verificar se são localmente convexos.

Caso $\epsilon>0$, é necessário procurar para cada simplexo $\sigma_{1}$ que possuem $p_{i}$ como um de seus vértices o simplexo $\sigma_{2}$ vizinho de $\sigma_{1}$ que não possua $p_{i}$ como vértice. Para cada dupla de simplexos é determinado o peso limitante, dado pela equação 4.16, que causa um flip entre $\sigma_{1}$ e $\sigma_{2}$, cada limitante será adicionado em ordem crescente em uma lista. Enquanto o primeiro peso da lista for menor que $w_{p_{i}}+\epsilon$ um flip ocorrerá com os respectivos simplexos. Quando um flip ocorre novos os simplexos são criados e devem ter os limitantes para seus flip calculados e adicionados em ordem na lista.

Análogo ao caso anterior, quando $\epsilon<0$ é necessário procurar para cada simplexo $\sigma_{1}$ que possuem $p_{i}$ como um de seus vértices o simplexo $\sigma_{2}$ vizinho de $\sigma_{1}$ e possua $p_{i}$ como vértice. Para cada dupla de simplexos será determinado o limitante de peso que causa um flip entre $\sigma_{1}$ e $\sigma_{2}$, estes pesos serão ordenados de forma decrescente em uma lista. Enquanto o primeiro peso da lista for maior que $w_{p_{i}}+\epsilon$ um flip ocorrerá com os respectivos simplexos. Quando um flip ocorre o novo simplexo de $p_{i}$ deve ter os limitantes para seu flip calculado e adicionados em ordem na lista de limitantes.

A seguir é apresentado um pseudo-código para este algoritmo:

1 Se $\epsilon>0$ então:

2 Procuras as duplas de simplexo que poderão ser flipadas.

3 Calcule para cada dupla o limitante de pesos.

4 Ordene em uma lista os pesos limitantes de forma crescente.

5 Enquanto o primeiro elemento da lista for menor que $w_{p_{i}}+\epsilon$ faça:

6 Remova o primeiro elemento da lista.

$7 \quad$ Realize um flip nesta dupla de simplexos.

8 Calcule o limitante para os novos simplexos e adicione na lista.

9 Se $\epsilon<0$ então: 
10 Procuras as duplas de simplexo que poderão ser flipadas.

11 Calcule para cada dupla o limitante de pesos.

12 Ordene em uma lista os pesos limitantes de forma decrescente.

13 Enquanto o primeiro elemento da lista for maior que $w_{p_{i}}+\epsilon$ faça:

14 Remova o primeiro elemento da lista.

15 Realize um flip nesta dupla de simplexos.

16 Calcule o limitante para os novos simplexos e adicione na lista.

\subsection{Calculando Pesos em Duas Dimensões}

Como descrito anteriormente, flips estão relacionados com mudanças nas relações de adjacência das faces do envelope superior dos planos associados aos pontos com peso. Tais mudanças ocorrem quando quatro planos se interceptam em um único ponto. Desta forma, para encontrar quando ocorrerá um flip, basta calcular quando os quatro planos definidos pelos quatro pontos do quadrilátero formado pela aresta e seus vértices opostos se interceptam em apenas um ponto. Sejam os pontos $\hat{p}_{i}, \hat{p}_{j}, \hat{p}_{k}, \hat{p}_{l}$ que formam os triângulos $p_{i} p_{l} p_{j}$ e $p_{i} p_{l} p_{k}$ temos as equações:

$$
\left\{\begin{array} { l } 
{ \eta _ { \hat { p } _ { i } } - \eta _ { \hat { p } _ { j } } = 0 } \\
{ \eta _ { \hat { p } _ { i } } - \eta _ { \hat { p } _ { k } } = 0 } \\
{ \eta _ { \hat { p } _ { i } } - \eta _ { \hat { p } _ { l } } = 0 }
\end{array} \Rightarrow \left\{\begin{array}{l}
-\left\|p_{i}\right\|^{2}+\left\|p_{j}\right\|^{2}+2\left\langle p_{i}, x\right\rangle-2\left\langle p_{j}, x\right\rangle+w_{p_{i}}-w_{p_{j}}=0 \\
-\left\|p_{i}\right\|^{2}+\left\|p_{k}\right\|^{2}+2\left\langle p_{i}, x\right\rangle-2\left\langle p_{k}, x\right\rangle+w_{p_{i}}-w_{p_{k}}=0 \\
-\left\|p_{i}\right\|^{2}+\left\|p_{l}\right\|^{2}+2\left\langle p_{i}, x\right\rangle-2\left\langle p_{l}, x\right\rangle+w_{p_{i}}-w_{p_{l}}=0
\end{array}\right.\right.
$$

Analisando as inequações 4.17 e 4.18 e o seus significados é possível escrever uma inequação para cada aresta de uma triangulação $T$, usando o teste descrito na seção 4.1.1, e montar um sistema de inequações, onde a solução nos dará um conjunto de pesos tal que associados aos pontos tornam a triangulação $T$ regular. Como este sistema não terá solução única define-se uma função objetivo e resolvendo com programação linear é possível encontrar uma solução única. Esta função objetivo pode ser por exemplo: 


$$
\text { minimizar : } f=\sum_{i=0}^{n} w_{p_{i}}
$$

onde $n$ é o número de pontos com peso da triangulação $T$.

Desta forma é possível encontrar os pesos que tornam uma triangulação regular, ou seja, apenas associando pesos aos pontos é possível reconstruir a malha original. Para isso uma nova função objetivo que maximiza o número de variáveis iguais a zero é apresentada da seguinte forma:

$$
\text { minimizar : } f=\sum_{i=0}^{n}\left(g\left(w_{p_{i}}\right)+w_{p_{i}}\right)
$$

onde,

$$
g\left(w_{i}\right)= \begin{cases}10^{10} & , \text { if } w_{p_{i}} \neq 0 \\ 0 & , \text { if } w_{p_{i}}=0\end{cases}
$$

Teorema 4.3.1. Seja T uma triangulação que possa ser escrita como uma triangulação regular, quando associado pesos aos seus pontos, então o sistema de inequações proposto encontra um conjunto de pesos que torne $T$ uma triangulação regular.

Demonstração 4.3.1. Supondo que para a triangulação $T$ exista um conjunto $W$ de pesos que a torne regular, ou seja $T$ é convexa e possua todas suas arestas localmente convexas quando associadas ao conjunto de pesos $W$. Como descrito, as equações 4.17 e 4.18 serão satisfeitas se as arestas forem localmente convexas, logo existe um conjunto de pesos que satisfaz todas as equações, pois os pesos de $W$ satisfazem todas as equações. Como independente da função objetivo, a programação linear sempre encontra uma solução, conclui-se que sempre que houver solução, o esquema proposto irá encontrá-la.

Para facilitar a compreensão deste problema e sua resolução é apresentado um conjunto de pontos $P$ com uma determinada triangulação $T$, a qual será encontrado um conjunto de pesos que associados a cada ponto torna a triangulação $T$ uma triangulação regular.

Seja $P$ um conjunto de sete pontos, sendo eles $\hat{p}_{1}=(-0.535,-0,36), \hat{p}_{2}=(-0.385,0.53)$, $\hat{p}_{3}=(0.46,0.31), \hat{p}_{4}=(-0.15,-0.38), \hat{p}_{5}=(-0.095,-0.245), \hat{p}_{6}=(-0.4,0.295) \mathrm{e}$ 
a)

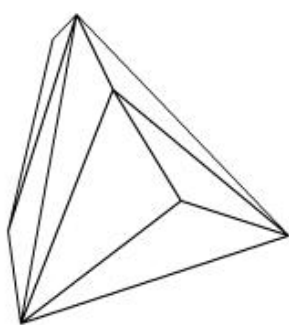

b)

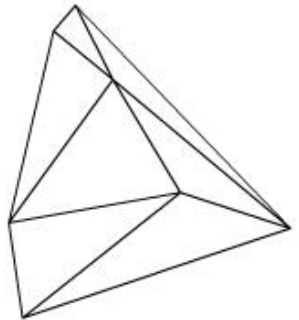

Figura 4.1: Em a) o conjunto $P$ e sua respectiva triangulação $T$, e em b) temos a triangulação Delaunay de $S$, note que a triangulação $T$ não é Delaunay.

$\hat{p}_{7}=(-0.4,0.295)$. Seja $T$ uma triangulação dos pontos de $P$ como representada na figura 4.1.

O objetivo é encontrar um conjunto de pesos $W=\left(w_{p_{1}}, w_{p_{2}}, w_{p_{3}}, w_{p_{4}}, w_{p_{5}}, w_{p_{6}}, w_{p_{7}}\right)$ que torne a triangulação $T$ uma triangulação regular, para isto é necessário escrever um sistema de inequações como descrito a seguir:

$$
\left\{\begin{array}{l}
-w_{p_{1}}+0.70565 w_{p_{5}}+0.19598 w_{p_{3}}+0.09836 w_{p_{2}}<+0.13849 \\
-w_{p_{1}}+0.59182 w_{p_{5}}-0.05336 w_{p_{3}}+0.46153 w_{p_{7}}<+0.05731 \\
-w_{p_{1}}+2.86107 w_{p_{7}}-1.34970 w_{p_{3}}-0.51137 w_{p_{2}}<-0.36469 \\
-w_{p_{1}}+0.36276 w_{p_{7}}+0.02104 w_{p_{2}}+0.61618 w_{p_{5}}<+0.07469 \\
-w_{p_{2}}+5.59484 w_{p_{7}}-2.63934 w_{p_{3}}-1.95550 w_{p_{1}}<-0.71315 \\
-w_{p_{2}}-17.2337 w_{p_{7}}+47.5064 w_{p_{1}}-29.2727 w_{p_{5}}<-3.54828 \\
-w_{p_{2}}+1.20271 w_{p_{6}}-0.88126 w_{p_{5}}+0.67854 w_{p_{1}}<-0.20540 \\
-w_{p_{3}}+11.0909 w_{p_{5}}-18.7402 w_{p_{1}}+8.64935 w_{p_{7}}<+1.07417 \\
-w_{p_{3}}+2.11978 w_{p_{7}}-0.74090 w_{p_{1}}-0.37888 w_{p_{2}}<-0.27020 \\
-w_{p_{5}}+1.41712 w_{p_{1}}-0.27773 w_{p_{3}}-0.13938 w_{p_{2}}<-0.19625 \\
-w_{p_{5}}+1.36038 w_{p_{4}}-0.81223 w_{p_{6}}+0.45184 w_{p_{2}}<+0.02978 \\
-w_{p_{5}}+1.36475 w_{p_{6}}-1.13472 w_{p_{2}}+0.76996 w_{p_{1}}<-0.23308 \\
-w_{p_{6}}+1.67486 w_{p_{4}}-1.23117 w_{p_{5}}+0.55630 w_{p_{2}}<+0.03667 \\
-w_{p_{7}}+0.34951 w_{p_{1}}+0.47174 w_{p_{3}}+0.17873 w_{p_{2}}<+0.12746
\end{array}\right.
$$

A resolução deste sistema por programação linear obtém a seguinte solução: 


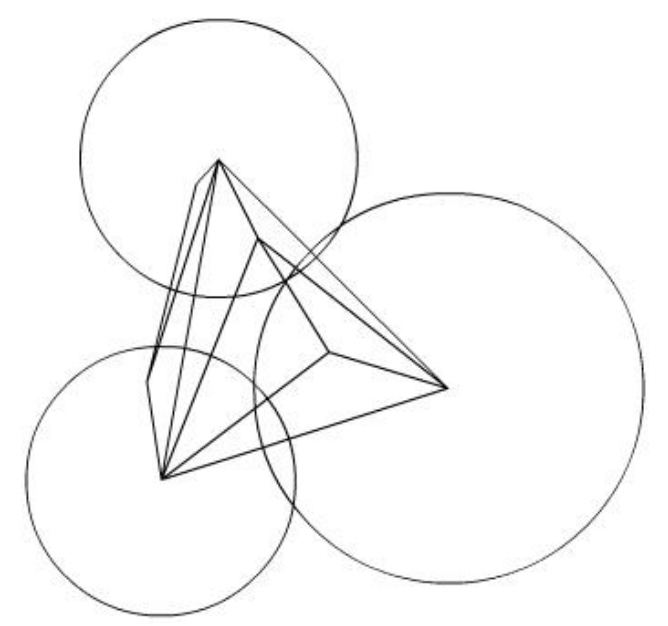

Figura 4.2: A triangulação de $P$ com os pesos $W$ associados a eles, e se obtém a triangulação $T$ como regular.

$$
W=\left[\begin{array}{c}
w_{p_{1}} \\
w_{p_{2}} \\
w_{p_{3}} \\
w_{p_{4}} \\
w_{p_{5}} \\
w_{p_{6}} \\
w_{p_{7}}
\end{array}\right]=\left[\begin{array}{c}
0.0 \\
0.10848 \\
0.22720 \\
0.0 \\
0.11458 \\
0.0 \\
0.0
\end{array}\right]
$$

Esta solução foi verificada e foi obtida a triangulação regular de $P$ idêntica à triangulação dada por $T$, ou seja, foi possível resolver o problema inverso para esse determinado conjunto e triangulação, como ilustra a figura 4.2 .

Para ilustrar, outro exemplo é mostrada na imagem 4.3 uma triangulação cujos cálculos resultaram nos pesos mostrados na triangulação da imagem 4.4, que é idêntica à triangulação inicial.

A tabela 4.1 mostra o tempo em segundos para gerar e resolver sistemas de inequações. Estes testes foram realizados em um computador com processador de $2.23 \mathrm{Mhz}$ e $1 \mathrm{~GB}$ de memória. 


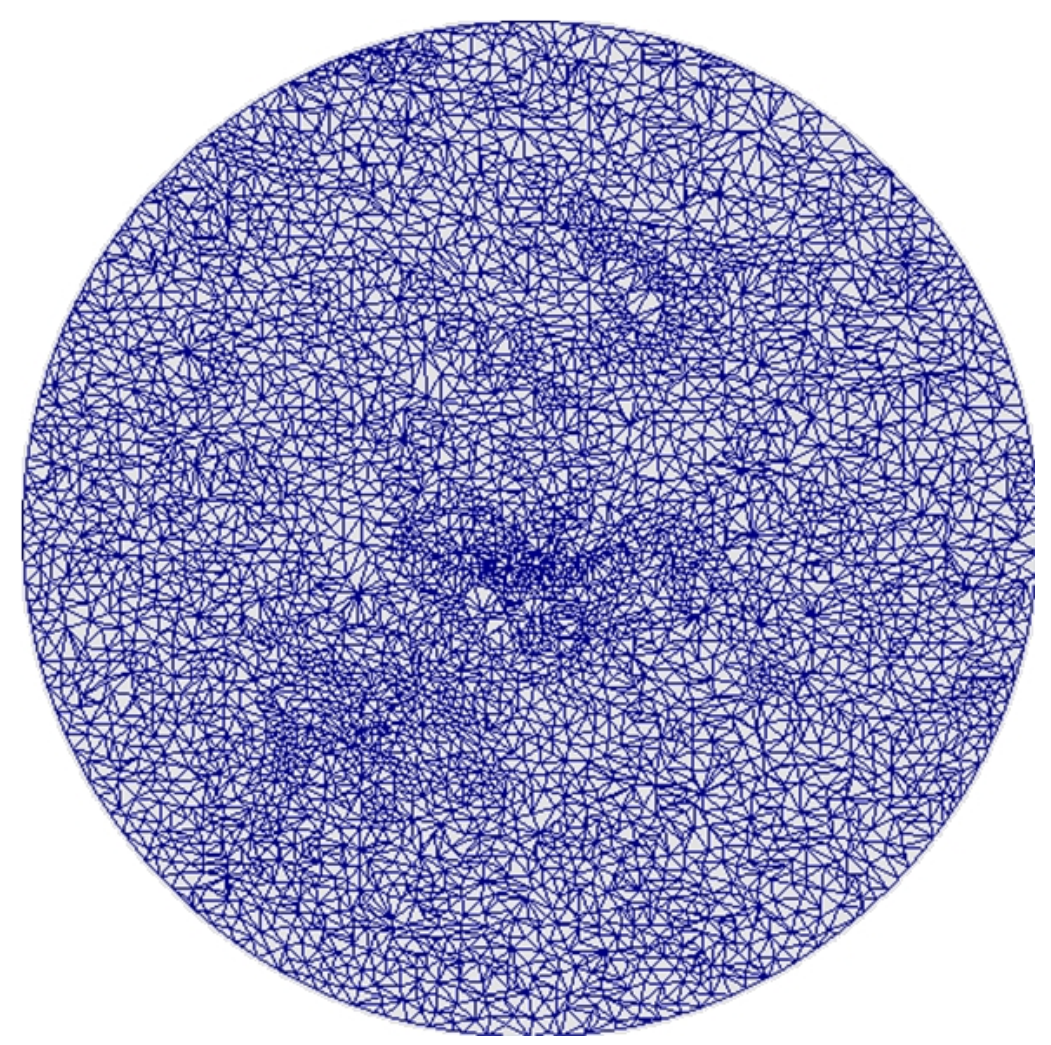

Figura 4.3: Uma triangulação cujas arestas não são localmente Delaunay.

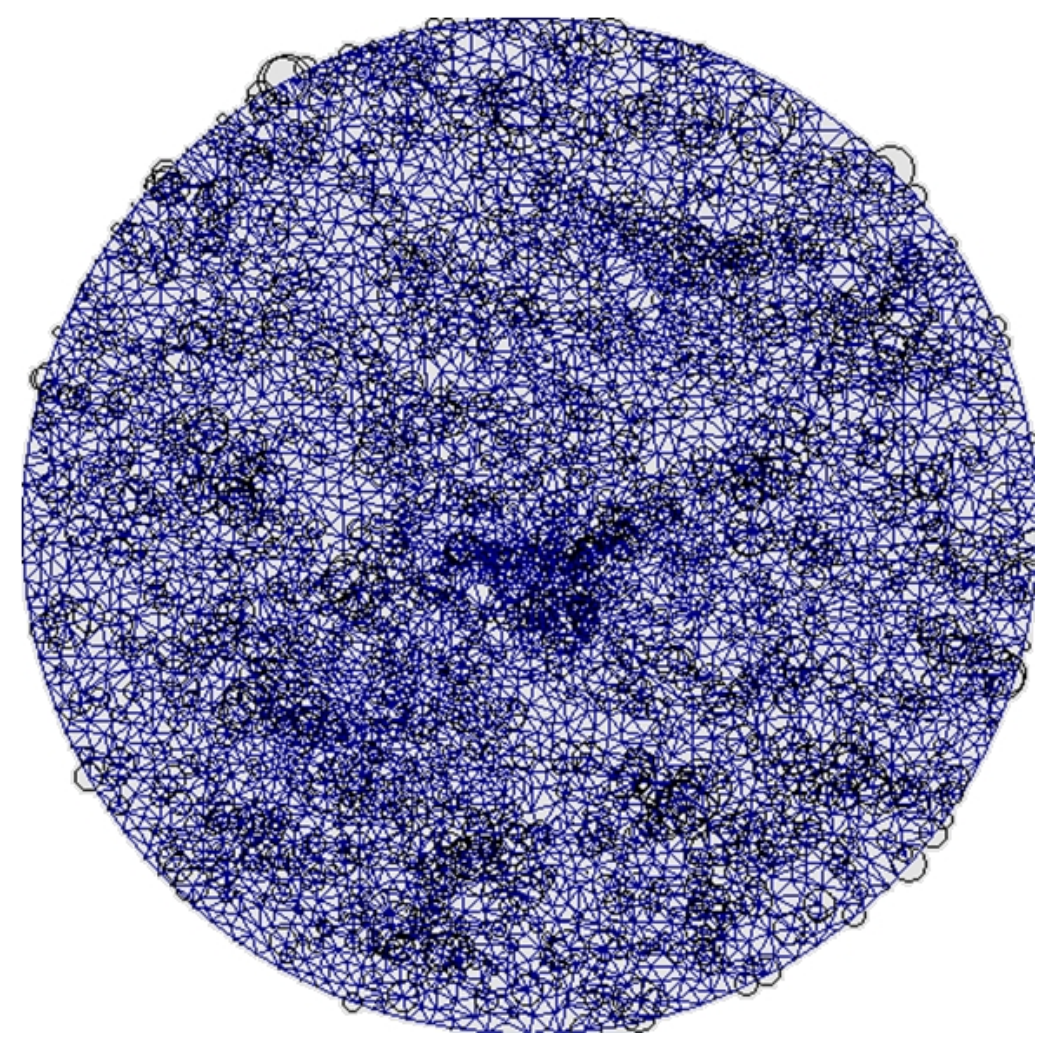

Figura 4.4: Triangulação regular e pesos dos vértices da imagem 4.3. 


\begin{tabular}{|l|l|l|l|}
\hline Triangulações & Gerar sistemas & Resolver usando 4.20 & Resolver usando 4.21 \\
\hline 10 de 400 pontos & $0,55 \mathrm{~s}$ & $9,02 \mathrm{~s}$ & $12,9142 \mathrm{~s}$ \\
\hline 10 de 2000 pontos & $8,63 \mathrm{~s}$ & $481,04 \mathrm{~s}$ & $626,8688 \mathrm{~s}$ \\
\hline 10 de 5000 pontos & $58,08 \mathrm{~s}$ & $12489,23 \mathrm{~s}$ & $17736,4298 \mathrm{~s}$ \\
\hline
\end{tabular}

Tabela 4.1: ilustra o tempo que nossa técnica levou para gerar e resolver os sistemas.

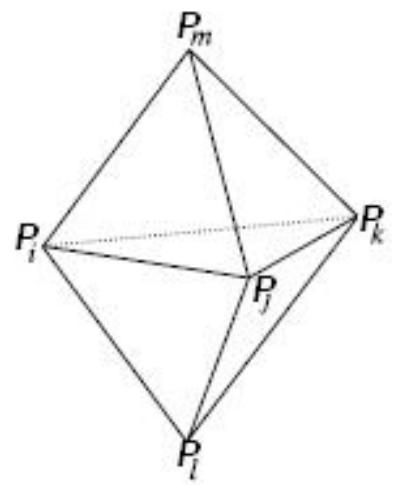

Figura 4.5: Configuração de triângulos estudada.

\subsection{Três Dimensões}

Assim como para o caso de duas dimensões, triangulações regulares em 3D podem ser calculadas usando o mesmo algoritmo. Desta forma, será descrito um algoritmo para prever quando um flip irá acontecer, considerando os seus pesos como incógnitas. Para prever quando um flip irá ocorrer é associado a cada vértice um hiperplano em $\mathbb{R}^{4}$ e a intersecção de quatro hiperplanos é um ponto cuja projeção é o ortocentro da ortosfera dos quatro vértices correspondentes. O hiperplano de um ponto $\hat{p}_{i}$ é dado por:

$$
\eta_{\hat{p}_{i}}(x)=2\left\langle v_{i}, x\right\rangle-\left\|p_{i}\right\|^{2}+w_{p_{i}}
$$

Seguindo o mesmo raciocínio do caso bidimensional, uma situação singular irá ocorrer quando os hiperplanos definidos pelos vértices das faces e os hiperplanos dos seus dois vértices opostos se encontrarem em um único ponto.

Sejam os pontos $\hat{p}_{i}, \hat{p}_{j}, \hat{p}_{k}, \hat{p}_{l}$ e $\hat{p}_{m}$ e os tetraedros $\hat{p}_{i} \hat{p}_{j} \hat{p}_{k} \hat{p}_{l}$ e $\hat{p}_{i} \hat{p}_{j} \hat{p}_{k} \hat{p}_{m}$, como mostra a figura 4.5, obtém-se as equações: 


$$
\left(\begin{array}{cccccccc}
A_{1} & B_{1} & C_{1} & 1 & -1 & 0 & 0 & 0 \\
A_{2} & B_{2} & C_{2} & 1 & 0 & -1 & 0 & 0 \\
A_{3} & B_{3} & C_{3} & 1 & 0 & 0 & -1 & 0 \\
A_{4} & B_{4} & C_{4} & 1 & 0 & 0 & 0 & -1
\end{array}\right)\left(\begin{array}{c}
x \\
y \\
z \\
w_{p_{i}} \\
w_{p_{j}} \\
w_{p_{k}} \\
w_{p_{l}} \\
w_{p_{m}}
\end{array}\right)=\left(\begin{array}{c}
D_{1} \\
D_{2} \\
D_{3} \\
D_{4}
\end{array}\right)
$$

onde,

$$
\begin{array}{ll}
A_{1}=2\left(x_{i}-x_{j}\right) & B_{1}=2\left(y_{i}-y_{j}\right) \\
A_{2}=2\left(x_{i}-x_{k}\right) & B_{2}=2\left(y_{i}-y_{k}\right) \\
A_{3}=2\left(x_{i}-x_{l}\right) & B_{3}=2\left(y_{i}-y_{l}\right) \\
A_{4}=2\left(x_{i}-x_{m}\right) & B_{4}=2\left(y_{i}-y_{m}\right) \\
& \\
C_{1}=2\left(z_{i}-z_{j}\right) & D_{1}=-\left\|x_{j}\right\|^{2}+\left\|x_{i}\right\|^{2} \\
C_{2}=2\left(z_{i}-z_{k}\right) & D_{2}=-\left\|x_{k}\right\|^{2}+\left\|x_{i}\right\|^{2} \\
C_{3}=2\left(z_{i}-z_{l}\right) & D_{3}=-\left\|x_{l}\right\|^{2}+\left\|x_{i}\right\|^{2} \\
C_{4}=2\left(z_{i}-z_{m}\right) & D_{4}=-\left\|x_{m}\right\|^{2}+\left\|x_{i}\right\|^{2}
\end{array}
$$

cuja solução é:

$$
w_{p_{i}}-w_{p_{j}}-w_{p_{k}}-w_{p_{l}}-w_{p_{m}}=\frac{P_{2} N_{1}-N_{2} P_{1}}{O_{2} N_{1}-N_{2} O_{1}}
$$

onde,

$$
\begin{array}{ccccc}
E_{1}=\frac{B_{2} A_{1}-A_{2} B_{1}}{A_{1}} & F_{1}=\frac{C_{2} A_{1}-A_{2} C_{1}}{A_{1}} & G_{1}=\frac{A_{1}-A_{2}}{A_{1}} & I_{1}=\frac{D_{2} A_{1}-A_{2} D_{1}}{A_{1}} & H_{1}=\frac{-A_{2}}{A_{1}} \\
E_{2}=\frac{B_{3} A_{1}-A_{3} B_{1}}{A_{1}} & F_{2}=\frac{C_{3} A_{1}-A_{3} C_{1}}{A_{1}} & G_{2}=\frac{A_{1}-A_{3}}{A_{1}} & I_{2}=\frac{D_{3} A_{1}-A_{3} D_{1}}{A_{1}} & H_{2}=\frac{-A_{3}}{A_{1}} \\
E_{3}=\frac{B_{4} A_{1}-A_{4} B_{1}}{A_{1}} & F_{3}=\frac{C_{4} A_{1}-A_{4} C_{1}}{A_{1}} & G_{3}=\frac{A_{1}-A_{4}}{A_{1}} & I_{3}=\frac{D_{4} A_{1}-A_{4} D_{1}}{A_{1}} & H_{3}=\frac{-A_{4}}{A_{1}} \\
N_{1}=\frac{F_{2} E_{1}-E_{2} F_{1}}{E_{1}} & O_{1}=\frac{G_{2} E_{1}-E_{2} G_{1}}{E_{1}} & P_{1}=\frac{I_{2} E_{1}-E_{2} I_{1}}{E_{1}} \\
N_{2}=\frac{F_{3} E_{1}-E_{3} F_{1}}{E_{1}} & O_{2}=\frac{G_{3} E_{1}-E_{3} G_{1}}{E_{1}} & P_{2}=\frac{I_{3} E_{1}-E_{3} I_{1}}{E_{1}}
\end{array}
$$




$$
\begin{array}{ll}
K_{1}=\frac{-E_{2}}{E_{1}} & J_{1}=\frac{H_{2} E_{1}-E_{2} H_{1}}{E_{1}} \\
K_{2}=\frac{-E_{3}}{E_{1}} & J_{2}=\frac{H_{3} E_{1}-E_{3} H_{1}}{E_{1}}
\end{array}
$$

Da mesma forma que para duas dimensões, para garantir que a face $p_{i} p_{j} p_{k}$ exista, os pesos devem satisfazer por exemplo:

$\left(O_{2} N_{1}-N_{2} O_{1}\right) w_{p_{i}}+\left(J_{2} N_{1}-N_{2} J_{1}\right) w_{p_{j}}+\left(K_{2} N_{1}-N_{2} K_{1}\right) w_{p_{k}}+N_{2} w_{p_{l}}-w_{p_{m}}>P_{2} N_{1}-N_{2} P_{1}$

Note que este problema pode ser resolvido, como em duas dimensões por meio de um problema de otimização linear, assim como deve ser usado o teste para determinar qual inequação usar quando $p_{i} p_{j} p_{k}$ for localmente Delaunay. Desta forma, será possível determinar um sistema de inequações para cada aresta e encontrar um conjunto de pesos que torne a triangulação regular.

\subsection{Triangulações não Convexas}

Até o momento foi discutido como tornar uma triangulação qualquer em uma triangulação regular. Neste momento é possível encontrar os pesos que tornem todas as arestas em arestas localmente convexa, porém se a triangulação inicial não for convexa nunca será possível torná-la em regular.

Caso a triangulação inicial não seja convexa será possível torná-la uma triangulação regular restrita onde o bordo é armazenado. Outra alternativa é adicionar novos triângulos de tal forma a tornar a triangulação convexa, possibilitando a utilização dos algoritmos para construção de triangulação regular, e depois removê-los da triangulação.

Para tornar uma triangulação convexa é feita uma lista $l$ com os vértices do bordo em sentido horário, por exemplo, para cada três vértices em seqüencia desta lista cria-se um triângulo, se este triângulo estiver no interior da triangulação, ele é descartado e outros três vértices da lista são testados. Caso este triângulo esteja no exterior da triangulação, ele será adicionado e o ponto que deixar de ser de bordo será removido de $l$. A imagem 4.6 ilustra este processo. 

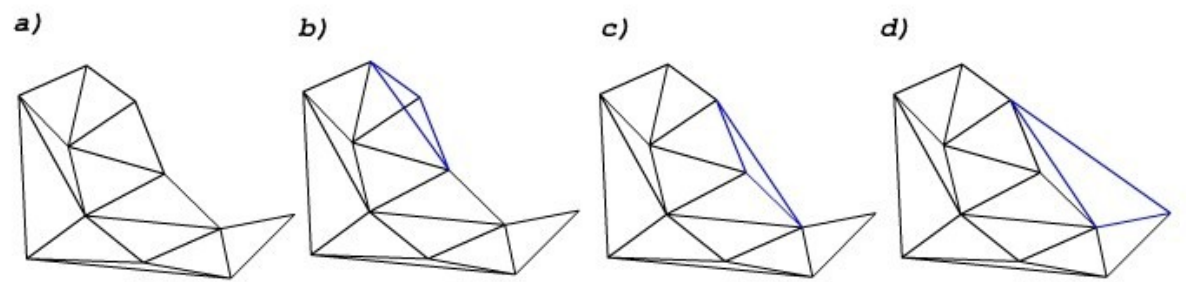

Figura 4.6: A imagem a) é uma triangulação não convexa, em b) foram escolhidos três vértices do bordo cujo triângulo formado por eles pertence ao interior da triangulação em c) e d) os triângulos formados pelos vértices do bordo pertencem ao exterior e serão adicionados à triangulação.

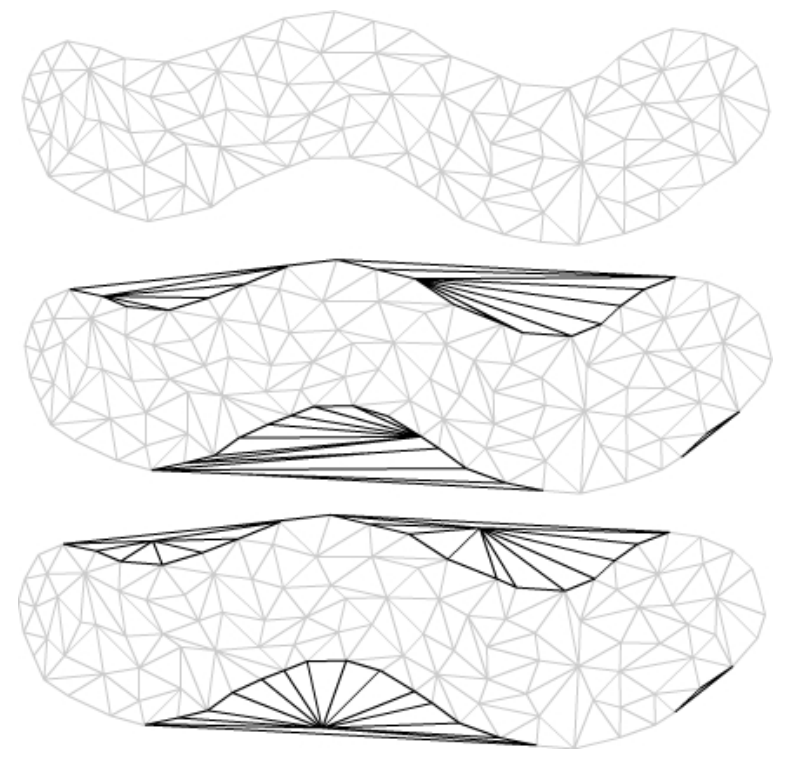

Figura 4.7: Nesta imagem, uma triangulação não convexa torna-se convexa utilizando o esquema descrito e pontos de controle são adicionados. Neste caso apenas quatro pontos de controles foram necessários.

Apesar da malha já ser convexa ainda será necessário armazenar todos os triângulos que foram adicionados neste processo para após calculada a triangulação regular serem removidos e assim obter a triangulação inicial.

A fim de minimizar o número de informações necessárias para remover os triângulos que foram adicionados, serão adicionados pontos de controle enquanto algum destes triângulos existirem, cada ponto será adicionado no interior de um destes novos triângulos com um flip 3-1 e enquanto for possível serão feitos flips $2-2$ dentre os novos triângulos. Desta forma, removendo todos os pontos de controle e todos seus triângulos da triangulação regular será obtida a triangulação inicial. A figura 4.7 ilustra este processo. 


\section{Capítulo 5}

\section{Aplicações}

Neste capítulo descrevemos três aplicações que desenvolvemos utilizando o mecanismo para determinar pesos, proposto no capítulo anterior. As aplicações são: compressão de malhas, nível de detalhes e morphing entre malhas. Estas aplicações apenas ilustram o potencial de aplicações quando uma malha triangular é transformada em triangulação regular, desta forma, não são feitos testes comparativos com outros métodos.

\subsection{Compressão de Malhas}

O problema de compressão de malha se divide em compressão de conectividade e compressão de coordenadas $[27,25]$. Usando o esquema de determinar pesos a uma triangulação qualquer será possível representar a conectividade pelos pesos, sendo desnecessário fazer a compressão de conectividade. Além da técnica descrita aqui muitas outras foram propostas como por exemplo [29], [14] e [28].

O trabalho de Bordignon [25] introduz um outro método de compressão de um conjunto de pontos que baseia-se na idéia de subdividir o espaço usando uma árvore BSP (binary space partition). Esta técnica usa informações geométricas para conseguir melhores resultados na compressão de malha.

Este algoritmo para compressão de ponto possui quatro características básicas, que serão enumeradas abaixo:

1. A subdivisão espacial baseia-se em cortar o espaço com planos ortogonais à direção 
principal, as regiões definidas pelos planos serão chamadas de células;

2. É necessário codificar a direção principal, que pode ser calculada usando mínimos quadrados, desta forma gerando um custo adicional que será distribuído para a maior quantia de pontos possível;

3. Para distribuir esse custo extra, o algoritmo reserva mais bits para codificar vértices próximos à raiz da BSP e menos bits para vértices mais próximos às folhas, ou seja, a quantização neste esquema é progressiva;

4. E finalmente o algoritmo desloca o plano que irá dividir o espaço em dois subespaços com a mesma quantia de pontos, para evitar a codificação de áreas vazias de pontos.

Na construção da árvore BSP temos que sua raiz contém todos os pontos. Esta técnica calcula, para uma célula, a matriz de covariância, depois o auto-vetor associado ao maior auto-valor, que representa a direção principal dos pontos contidos nesta célula. Finalmente, divide o conjunto de pontos em duas partes com a mesma quantidade de pontos, através da mediana dos pontos projetados na direção principal. Desta forma, pode-se obter os ângulos que definem a normal do plano, através da direção principal, e o deslocamento do plano que divide o conjunto de pontos no meio.

Com o plano de subdivisão calculado é preciso codificar suas coordenadas, ângulos $\phi$ e $\psi$ que definem sua normal e o deslocamento $\alpha$, como ilustra a figura 5.1. O número de bits usados para codificar estas informações depende do número de pontos presentes na célula dada. Por exemplo, se existirem $r$ pontos em uma célula, o número de bits codificados é dado por $n b=\log _{2}(r)-k$, onde $k$ varia de acordo com o parâmetro que está sendo codificado (k é 7, 5 e 3 para $\phi, \psi$ e $\alpha$ respectivamente), ou seja, quanto mais pontos houver em uma célula mais bits serão usados para codificar as coordenadas de um plano. Note que se houver poucos pontos, $n b$ pode ser zero ou negativo, para evitar que esse erro limitasse a quantia mínima de bits para codificar uma coordenada.

O último passo do algoritmo consiste em quantizar os parâmetros usando uma função aritmética de codificação progressiva. Esta técnica foi comparada com outros métodos e para todos os conjuntos de dados testados, ela obteve uma melhor taxa de compressão, 

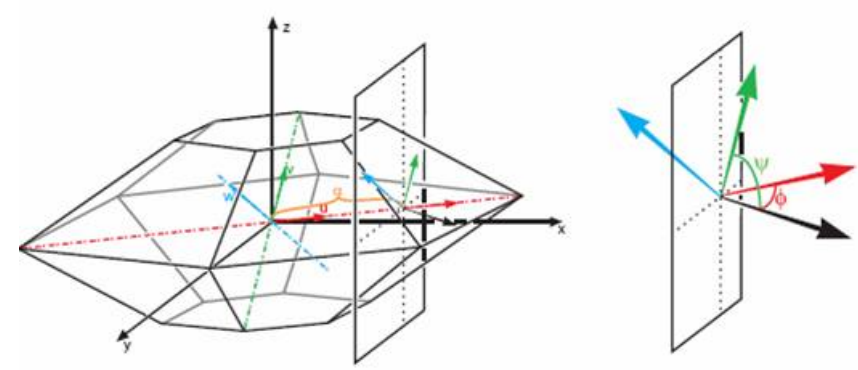

Figura 5.1: Plano de subdivisão de uma região, imagem retirada de [25].

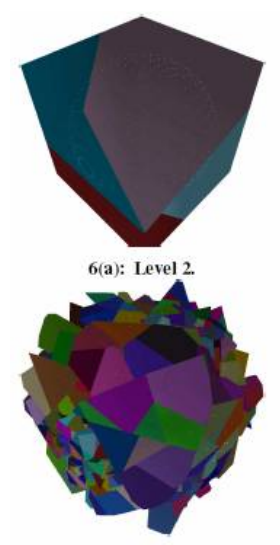

6(e): Level 14.

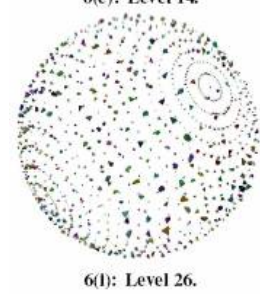

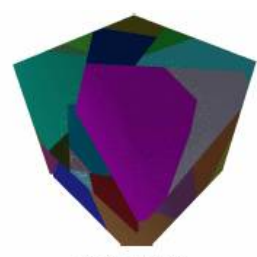

6(b): Level 5 .

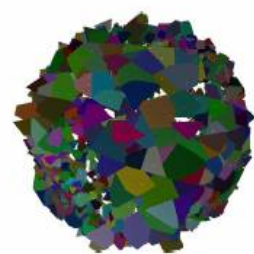

6(f): Level 17 .

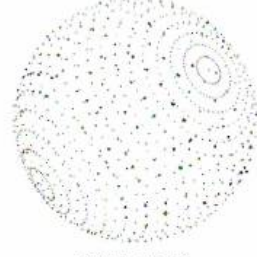

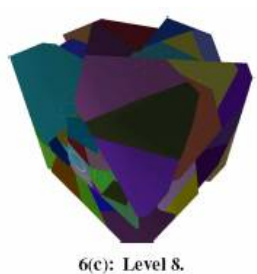

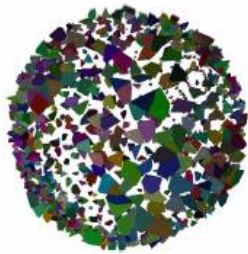

6(g): Level 20.

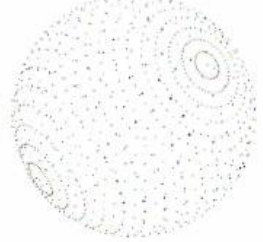

6(k): Level 32 .
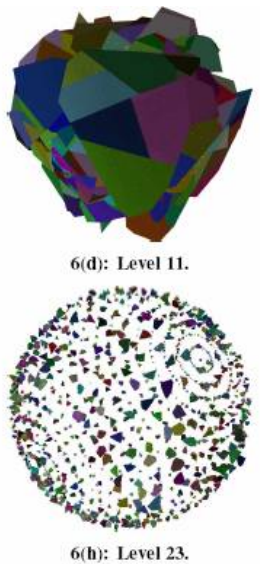

6(h): Level 23 .

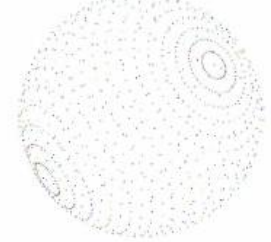

6(1): Level 35 .

Figura 5.2: Exemplo com alguns níveis de subdivisão gerados pela BSP, imagem retirada de $[25]$

segundo os autores cerca de $15 \%$ mais eficiente. A figura 5.2 contém um exemplo com alguns passos deste algoritmo.

Utilizando deste esquema de compressão de coordenadas, ou qualquer outro esquema, ainda é necessário um esquema de compressão de conectividade. Desta forma, será utilizado para compressão de conectividade o esquema para encontrar pesos em uma triangulação. Uma vez transformada a triangulação em regular a conectividade pode ser obtida usando algoritmos para a construção de triangulações regulares.

Para que o esquema de triangulações regulares possa ser usado para realmente comprimir a malha é necessário associar pesos a um número pequeno vértices, caso contrário, se cada ponto da malha receber um número real teríamos em $\mathbb{R}^{3}$ um acréscimo de $25 \%$ 
das informações. Como vimos, para determinar os pesos de uma triangulação usamos uma função objetivo como 4.20 onde $n$ é o número de pontos com peso da triangulação T. Como a função minimiza a soma dos pesos é de esperar que vários dos pesos sejam iguais a zero, porém eventualmente todos os pesos poderiam ser diferentes de zero.

Uma outra função objetivo 4.21 que maximiza o número de variáveis iguais a zero pode ser apresentada, tornando o resultado da compressão mais eficaz do que a equação anterior.

Ainda assim em um caso particular poderia ocorrer que poucos pontos fossem iguais a zero caindo no mesmo problema de armazenar muitos pesos para a triangulação. Propomos então uma terceira função objetivo que maximiza o número de variáveis iguais, ou seja, minimiza os possíveis valores que os pesos podem assumir:

$$
\text { minimizar : } f=\sum_{i=0}^{n} \sum_{j=0}^{n}\left(w_{p_{i}}-w_{p_{j}}\right)^{2}
$$

Note que quanto mais eficiente a função objetivo mais complexa ela se torna, nesta ultima, a função deixou de ser linear, dificultando muito a resolução do sistema de inequações.

Após obter os pesos com a função objetivo 5.1, o esquema de compressão pode funcionar primeiro ordenando os vértices da malha agrupando os pontos que tenham pesos iguais em uma lista $l$, depois criar uma tabela com os possíveis pesos e o número de pontos que possuem este peso de tal forma que o primeiro valor desta tabela indique que os $n$ primeiros pontos da lista $l$ tenham o peso $w_{1}$, o segundo valor desta tabela indique que os próximos $m$ pontos da lista $l$ tenham o peso $w_{2}$, e assim por diante.

Em uma primeira análise da compressão é possível concluir que no pior caso, onde para todos os pontos são associados pesos diferentes, o número de dados adicionais corresponde a no máximo $25 \%$ do número total de dados (pontos e pesos), pois, são necessários três valores reais para as posições dos pontos e um para o peso. Porém, em nossos testes, a função objetivo 4.20 apresenta em média $30 \%$ dos pesos iguais a zero, implicando em apenas 17,5\% do total dados necessários. Já a função objetivo 4.21 apresenta em média $65 \%$ dos pesos iguais a zero, implicando, $8.75 \%$ do total de dados. 
Este esquema pode ser aplicado para comprimir malhas em duas ou três dimensões. Porém, para malhas de superfícies uma vez descartada a topologia não é mais possível reconstruir a triangulação original, pois os algoritmos não funcionam em codimensão diferente de zero. Neste caso o método realiza compressão com perdas, por isso não é possível recuperar a malha original.

Caso a triangulação superficial de um conjunto $P$ fosse o bordo do fecho convexo de $P$ seria possível usar o algoritmo de construção de uma triangulação regular em três dimensões para obter a malha inicial, sendo assim aplicável o esquema proposto, porém este caso particular é muito limitado e não representa problemas reais.

\subsection{Nivel de Detalhes}

Nível de detalhes de uma malha é, em poucas palavras, obter malhas simplificadas a partir de uma malha mais complexa. Este problema pode ser aplicado em diversas áreas, por exemplo, em mecânica de fluidos quando a malha é muito complexa pode ser simplificada em vários níveis e o resultado de uma simulação de nível $n$ será usada como aproximação inicial para a simulação no nível $n-1$. Esta aplicação foi desenvolvida com a colaboração de Dietrich e Comba.

Nível de refinamento é um problema que pode se beneficiar da teoria aqui desenvolvida. Dada uma malha inicial, deseja-se obter uma malha simplificada em um dado nível de detalhes. Primeiramente são escolhidos vértices ou regiões que serão preservadas o máximo possível na triangulação das malhas simplificadas, as demais regiões são simplificadas baseadas em algum critério, por exemplo, remover vértices de forma uniforme, ou seja, proporcional à densidade de vértices locais.

A contribuição que triangulações regulares traz a este problema é que a obtenção de uma topologia correta de uma malha simplificada é automaticamente obtida com o algoritmo para o cálculo de triangulações regulares.

A idéia é parametrizar a malha original via um algoritmo de parametrização de superfícies trianguladas.

Para construir as malhas refinadas será parametrizada a superfície em um domínio 

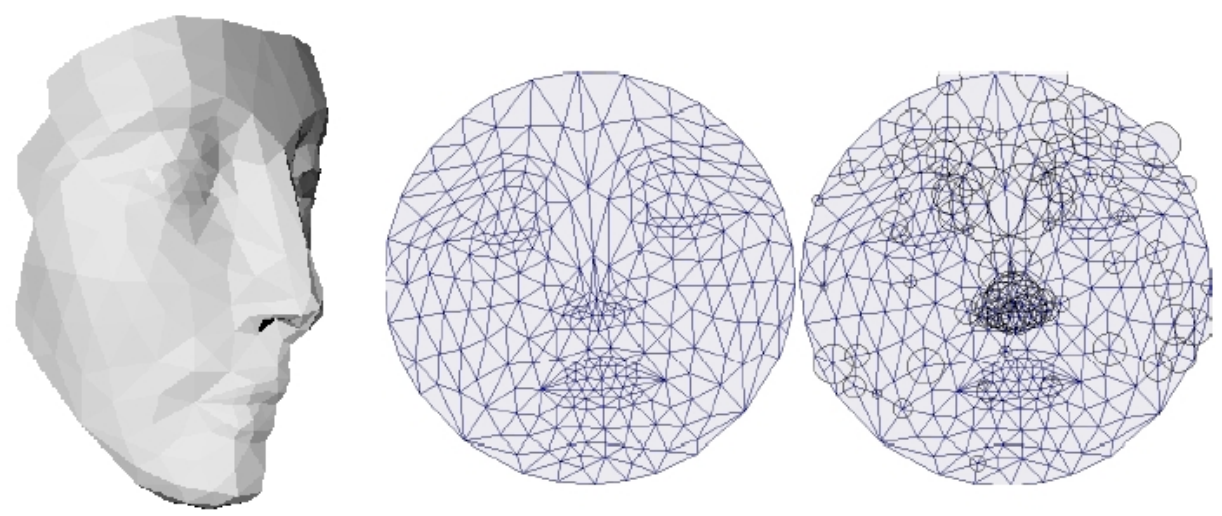

Figura 5.3: Na primeira imagem temos uma superfície homeomorfa a um disco, que na imagem segundo é parametrizada em um domínio convexo bidimensional, cujos pesos que a tornam uma triangulação regular São mostrados na terceira imagem.

convexo bidimensional, tal parametrização simplificará os cálculos. Nesta malha parametrizada serão calculados os pesos que a tornem uma triangulação regular e as simplificações serão feitas também na parametrização.

Parametrizar consiste em encontrar um mapeamento um a um de uma superfície para uma malha bidimensional. É necessário que a superfície seja homeomorfa a um disco, pois de outra maneira não será possível parametrizar em um disco. A imagem 5.3 ilustra um exemplo de parametrização de superfície. Neste trabalho não será descrito em mais detalhes o esquema de parametrização pois este foi utilizado apenas como uma ferramenta.

Para remover um vértice basta alterar seu peso para um valor muito pequeno, tendendo a $-\infty$, e automaticamente o algoritmo para triangulações regulares irá removê-lo pois este ponto será redundante (se este não for vértice do bordo). Caso seja vértice do bordo alterar seu peso para $-\infty$ fará com que este vértice não possua nenhum triângulo flipável, podendo ser removido sem prejudicar a triangulação.

Obtida a triangulação em um nível de refinamento na malha parametrizada, basta reproduzir sua topologia na malha superficial e será obtido o nível de refinamento desejado. A imagem 5.4 mostra alguns resultados. 

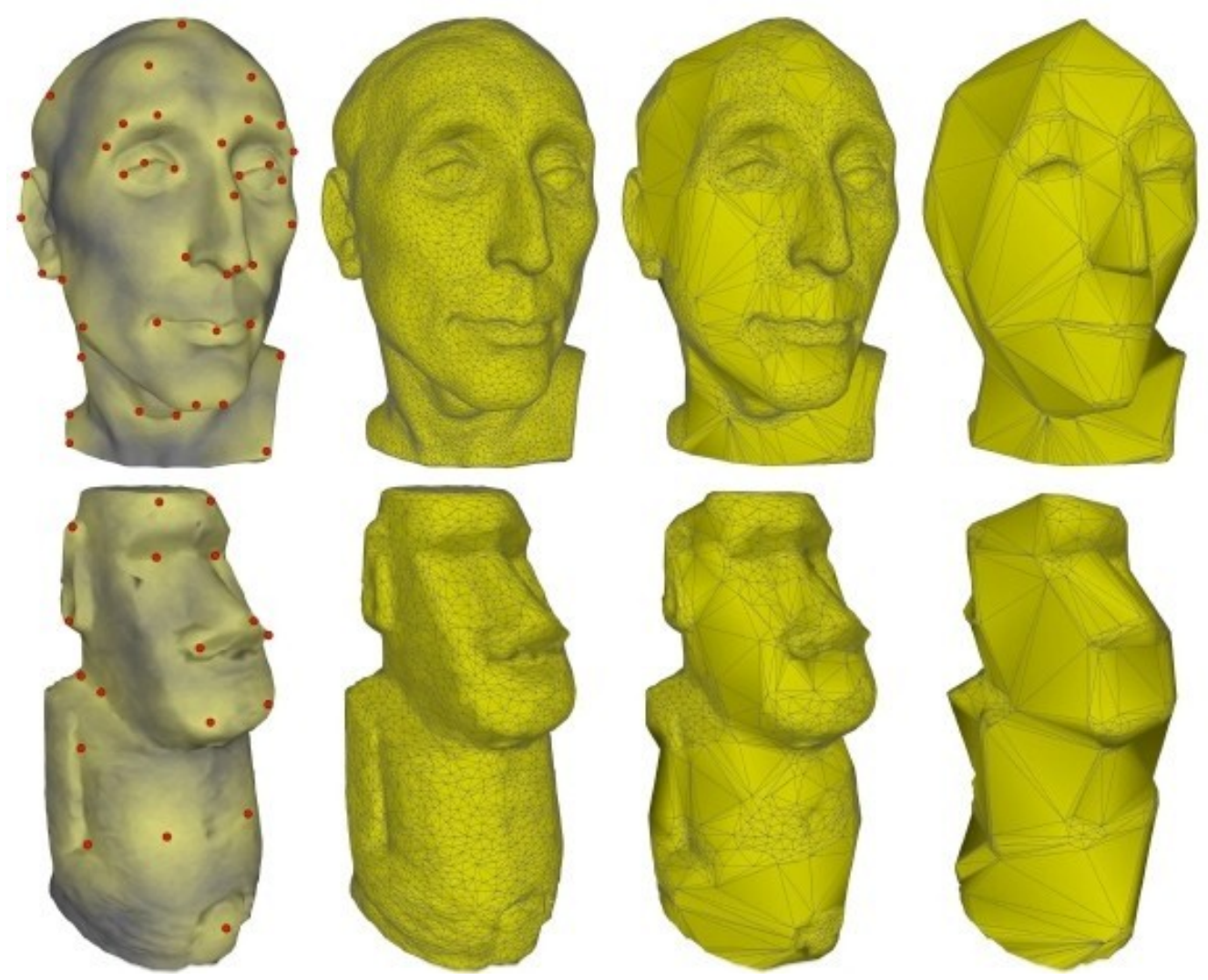

Figura 5.4: Exemplo de resultados de níveis de refinamento obtidos com este esquema, em ambos os casos os pontos em destaque foram escolhidos pelo usuário.

\subsection{Morphing de Malhas}

Morphing de malhas consiste em determinar malhas intermediárias válidas que transformem uma malha inicial $A$ em uma malha final $B$. A maioria dos algoritmos de morphing utiliza interpolação de vértices entre as malhas iniciais e finais para obter os vértices intermediários. Porém a dificuldade desta aplicação está em construir malhas intermediárias válidas. Desta forma existem muitas alternativas para obter a conectividade das malhas intermediárias [18, 21, 24].

Nesta seção será mostrada uma forma de utilizar triangulações regulares para obter as malhas intermediárias, estas, assim como em nível de refinamento, serão obtidas parametrizando as malhas superficiais (malhas formada por triângulos). Nesta aplicação também tivemos a colaboração de Dietrich e Comba. As parametrizações serão transformadas em triangulações regulares e, posteriormente, todas as alterações que forem feitas nas malhas parametrizadas serão reproduzidas na superfície, criando as malhas 


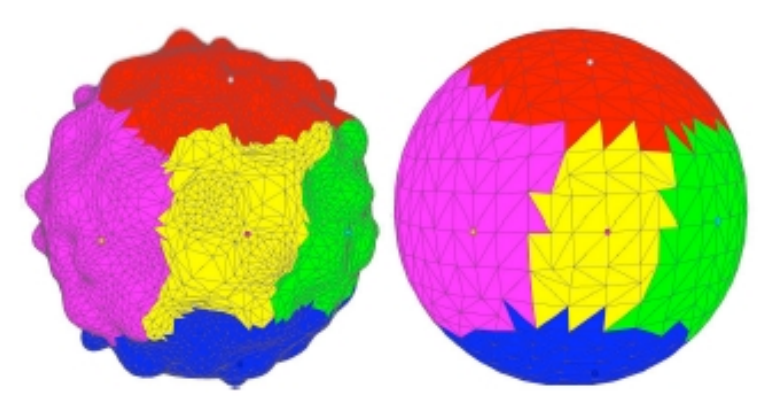

Figura 5.5: Exemplo de como dividir malhas em regiões e correspondê-las entre duas malhas.

intermediárias.

Neste caso, a superfície será dividida em pequenas regiões e as transformações serão feitas entre uma região da malha $A$ para outra região da malha $B$, cada região será parametrizada e os pesos que a tornem regular serão calculados. O morphing será feito entre estas malhas, que agora são triangulações regulares. O algoritmo para morphing entre malhas envolve os seguintes passos:

1. Correspondência de regiões: o morphing é realizado entre um par de regiões entre a malha inicial e a malha final.

2. Consistência do bordo: vértices pertencentes a duas regiões (vértices do bordo) precisam de tratamento especial para preservar continuidade.

3. Correspondência de vértices: mapeamento entre os vértices e pesos dados pela triangulação regular além das informações para continuidade do bordo.

4. Criar malhas intermediárias: produzir uma malha para qualquer vértice intermediário.

A malha inicial e a malha final devem ser divididas em um número igual de regiões para que seja possível uma transformação um a um. Além disso, também é necessário ser definido pares de regiões correspondentes sendo uma região de $A$ e uma de $B$, como ilustra a figura 5.5 .

A vantagem de utilizar regiões menores no processo de morphing é que os cálculos dos pesos se torna mais ágil e também a escolha das regiões correspondentes permite que em 

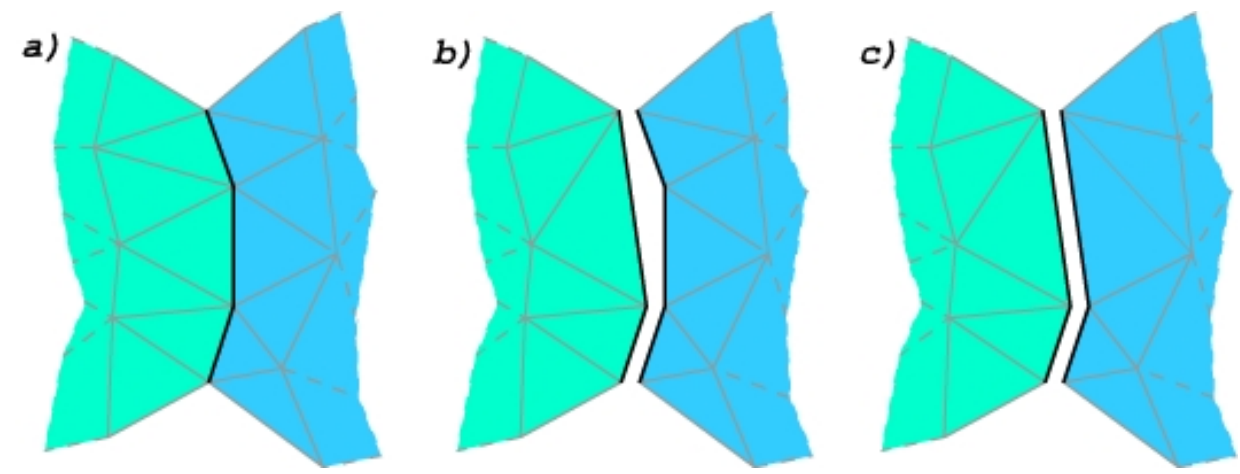

Figura 5.6: Em a) uma triangulação foi dividida em duas regiões, em b) um vértice do bordo foi removido de uma das regiões criando um buraco em c) o vértice foi removido simultaneamente em ambas as regiões.

uma transformação as partes similares em na malha inicial se transformem nas mesmas partes da malha final. Por exemplo, se as malhas $A$ e $B$ forem faces de pessoas, as regiões garantem a transformação de olho direito de $A$ em olho direito de $B$, nariz de $A$ em nariz de $B$ ou, se for o caso, seria possível transformar uma orelha de $A$ na boca de $B$.

As regiões criadas na malha $A$ em geral não possuem a mesma quantidade de vértices que suas correspondentes regiões na malha $B$, ou seja, durante o processo de morphing vértices devem ser adicionados ou removidos. Neste caso, uma região inicial será a região que possua mais vértices dentre ela e sua correspondente região, desta forma apenas será necessário remover vértices da região inicial; estes vértices serão removidos exatamente como foram removidos em nível de refinamento.

Ao remover um vértice que pertença ao interior de uma região nenhum problema é criado, porém, ao remover um vértice de bordo é necessário atenção, pois, este pertence a pelo menos uma outra região e deve ser removido simultaneamente, caso contrário, cria-se uma inconsistência nas malhas intermediárias, como ilustra a figura 5.6. O mesmo cuidado deve ser tomado ao tornar um vértice do interior em vértice do bordo.

Para os vértices que são mapeados em outros vértices (não são removidos ou inseridos) é feita uma transformação linear um a um. Por exemplo, seja o vértice $p$ de uma região de origem e $q$ um vértice da região final correspondente, os vértices intermediários $v(t)$ podem ser obtidos com a equação $v(t)=t q+(1-t) p \operatorname{com} 0<t<1$. Se $t=0$ então $v$ é vértice da malha inicial, se $t=1$ então $v$ é vértice da malha final. Caso necessário, a função que determina um vértice intermediário pode ser alterada e o resultado do morphing será 
diferente.

Com os pesos é possível utilizar o mesmo esquema de transformação, porém, com vértices que serão removidos, utilizar a mesma transformação pode fazê-los serem todos removidos com um $t$ muito pequeno, enquanto os demais vértices quase não sofreram alterações, causando uma alteração brusca no morphing em poucos passos. Neste caso, uma função de convergência mais lenta pode ser usada, como por exemplo, $w(t)=t^{2} w_{f}+$ $(1-t) w_{p}$ com $0<t<1$, onde $w_{p}$ é o peso de um vértice que será removido e $w_{f} \rightarrow-\infty$.

Dadas as malhas parametrizadas serão calculados os pesos que a tornem regular e suas malhas intermediárias podem ser obtidas como descrito acima. Os vértices intermediários da superfície podem ser encontrados assim como os vértices parametrizados e a topologia será reconstruída copiando a topologia da respectiva malha intermediária projetada. A imagem 5.7 mostra alguns resultados.

Note que as malhas intermediárias são sempre obtidas calculando a triangulação regular do conjunto de vértices e pesos intermediários, e por este motivo, sempre será obtida uma malha intermediária independente da escolha para os vértices correspondentes entre as malhas. 


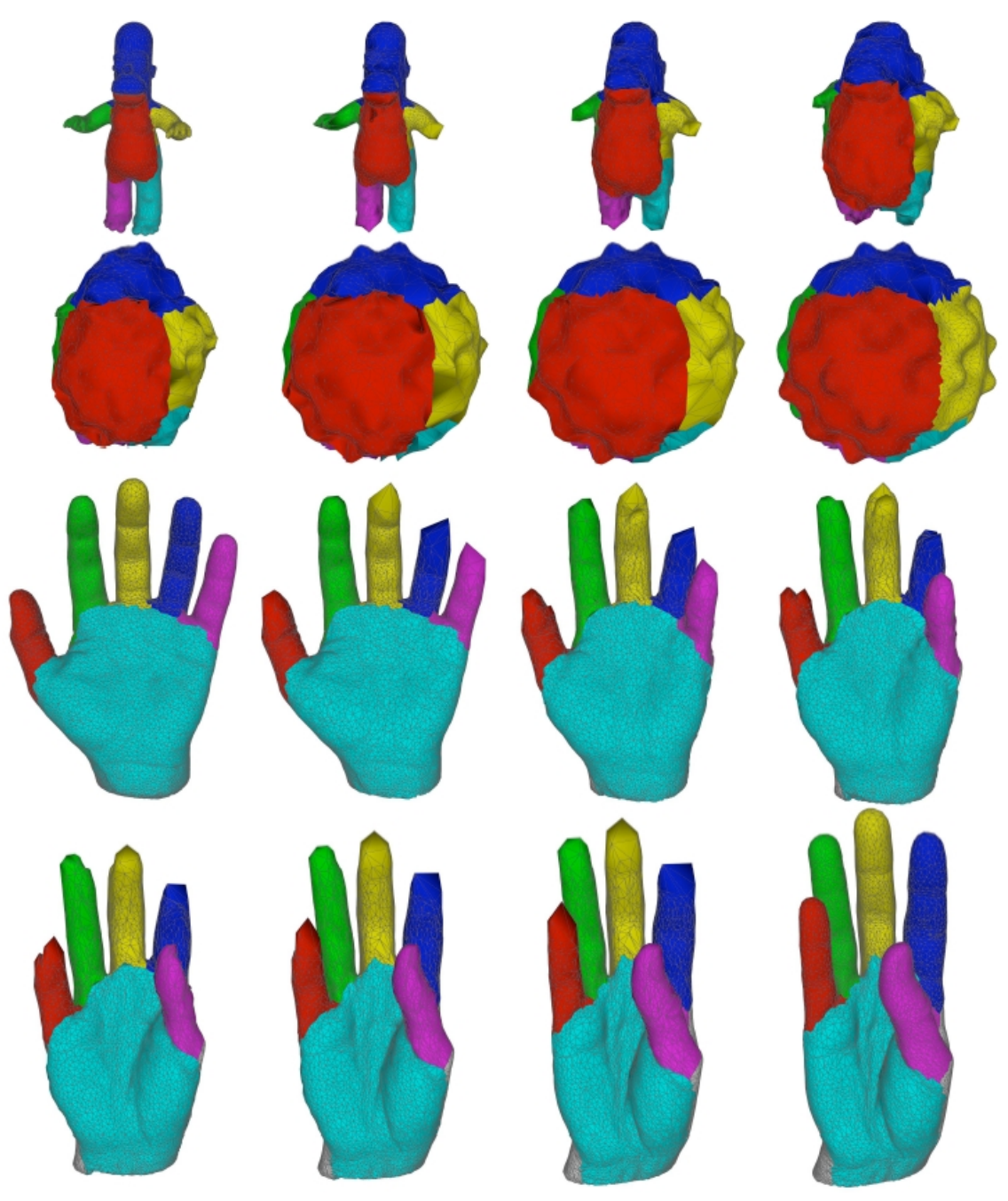

Figura 5.7: Dois exemplos de morphing entre malhas obtidos através de interpolações entre triangulações regulares. 



\section{Capítulo 6}

\section{Conclusões}

Como proposto inicialmente, um algoritmo para encontrar pesos que tornem uma triangulação qualquer em regular foi apresentado e demonstrado que existindo solução ele a encontra. Com os testes realizados podemos notar que a resolução do sistema de inequações parece satisfazer o problema de encontrar os pesos com eficiência, pois todos os testes obtiveram solução.

Como o esquema para o cálculo de pesos gera um sistema de inequações que é resolvido com programação linear, existe a liberdade de alterar a função objetivo ou utilizar minimização inteira $[16,26]$, pode-se ainda utilizar variáveis canalizadas de acordo com a necessidade da aplicação. É claro que a adição de restrições deve ser feita com cuidado para não tornar o problema infactível.

Nos testes realizados ficou evidente que a quantidade de pesos iguais a zero tende a ser grande e várias funções objetivos podem ser propostas com a finalidade de minimizar a quantia de dados necessários para reconstruir a malha original, o que garantiria bons resultados quando aplicado em compressão de malha. Infelizmente não foi possível levar esta aplicação adiante para o caso de superfícies devido à inexistência de um algoritmo que reconstrua superfície a partir de pontos e pesos.

Por outro lado, o trabalho descreveu como triangulações regulares podem ser úteis em nível de refinamento e morphing de malhas. Em nível de refinamento, uma vez com os pesos determinados, é necessário apenas alterar os pesos dos pontos que serão removidos para um valor muito baixo e automaticamente se obtém uma malha simplificada. Em 
morphing de malhas utilizar triangulações regulares foi muito útil pois independente da escolha da transformação entre os pontos sempre é possível encontrar uma malha intermediária, apesar de que uma boa escolha para a transformação apresenta um resultado mais agradável visualmente.

Apesar de bons resultados com estas aplicações não foram feitos testes comparativos com outros métodos, pios, o objetivo foi ilustrar o potencial da metodologia proposta para obter a topologia dos modelos nas aplicações. 


\section{Referências Bibliográficas}

[1] N. Amenta; and R. K. Kolluri. The medial axis of a union of balls. Computational Geometry: Theory and Applications, pages 25-37, 2001.

[2] F. Aurenhammer. Power diagrams: Properties, algorithms and applications. SIAM J. Comput., 16:78-96, 1987.

[3] B. Chazelle; and H. Edelsbrunner. An improved algorithm for constructing kth-order voronoi diagrams. In Symposium on Computational geometry, pages 228-234. ACM, 1985.

[4] L. Paul Chew. Guaranteed-quality mesh generation for curved surfaces. In Proceedings of the ninth annual symposium on Computational geometry, pages 274-280. ACM, 1993.

[5] N. Amenta; S. Choi; and R. K. Kolluri. The power crust, unions of balls, and the medial axis transform. Computatinal Geometry, 19:127-153, 2001.

[6] M. de Berg; M. van Kreveld; M. Overmars; and O. Schwarzkopf. Computational geometry - algorithms and applications. 1997.

[7] H. Edelsbrunner. Deformable smooth surface design. Discrete and Computational Geometry, 21:87-115, 1999.

[8] H. Edelsbrunner; and R. Seidel. Voronoi diagrams and arrangements. pages 25-44, 1986.

[9] H. Edelsbrunner; and S. Shah. Incremental topological flipping works for regular triangulations. pages 223-241, 1992. 
[10] J. Erickson. Dense point sets have sparse delaunay triangulations or ... but not too nasty. Discrete and Computational Geometry, pages 83-115, 2005.

[11] M. A. Facello. Implementation of a randomized algorithm for delaunay and regular triangulations in three dimensions. Computer Aided Geometric Design, pages 349$370,1995$.

[12] S. W. Cheng; Tamal K. Dey; H. Edelsbrunner; M. A. Facello; and S. H. Teng. Sliver exudation. pages 1-13, 2000.

[13] S. Fortune. A sweepline algorithm for voronoi diagrams. In Proceedings of the second annual symposium on Computational geometry, pages 313-322. ACM, 1986.

[14] B. Kronrod; and C. Gotsman. Optimized triangle mesh compression using prediction trees. The Eighth Pacific Conference, pages 406-407, 2000.

[15] N. Kruithof; and G. Vegter. Approximation by skin surfaces. pages 86-95, 2003.

[16] E. L. Lawler; and D. E. Wood. Branch-and-bound methods: A survey. Operations Research, pages 699-719, 1966.

[17] C. L. Lawson. Generation of a triangulation grid with application to contour plotting. In Technology Memorandum 299, California Institute of Technology Jet Propulsion, pages 123-142. ACM, 1972.

[18] M. Ahn; S. Lee; and H. Seidel. Connectivity transformation for mesh metamorphosis. In Proceedings of the 2004 Eurographics, pages 77-84. ACM, 2004.

[19] M. Melkemi; and M. Djebali. Weighted a-shape: a descriptor of the shape of a point set. Pattern Recognition, pages 1159-1170, 2001.

[20] J. O'Rourke. Computational geometry in c (cambridge tracts in theoretical computer science). 1998.

[21] J. Parus; and I. Kolingerovà. Morphing of meshes with attributes. In Proceedings of the 20th spring conference on Computer graphics, pages 73-81. ACM, 2004. 
[22] M. Vigo; Núria Pla; and J. Cotrina. Regular triangulations of dynamic sets of points. Computer Aided Geometric Design, 19:127-149, 2002.

[23] A. Aggarwal; L. Guibas; J. Saxe; and P. Shor. A linear time algorithm for computing the voronoi diagram of a convex polygon. pages 39-45, 1987.

[24] A. W. F. Lee; D. Dobkin; W. Sweldens; and P. Schröder. Multiresolution mesh morphing. pages 343-350. ACM, 1999.

[25] A. Bordignon; T. Lewiner; H. Lopes; G. Tavares; and R. Pereira. Point set compression through BSP quantization. In 19th Brazilian Symposium on Computer Graphics and Image Processing, pages 229-238, 2006.

[26] J. A. Tomlin. An improved branch-and-bound method for integer programming. Operations Research, pages 1070-1074, 1971.

[27] C. Touma; and C. Gotsman. Triangle mesh compression. Proceedings of the 24th Conference on Graphics Interface, pages 26-34, 1998.

[28] T. Lewiner; M. Craizer; H. Lopes; S. Pesco; L. Velho; and E. Medeiros. Gencode: Geometry-driven compression in arbitrary dimension and co-dimension. SIBGRAPI 2005. 18th Brazilian Symposium, pages 249-256, 2005.

[29] Xianfeng Wu; and Dehua Li. Triangle mesh compression and simplification. Machine Graphics and Vision International Journal, pages 475-488, 2002. 